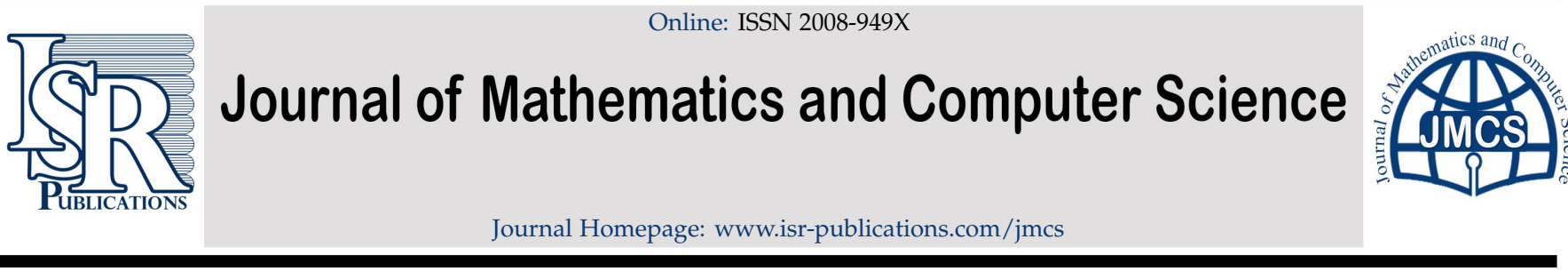

\title{
Degree of approximation for bivariate extension of blending type q-Durrmeyer operators based on Pólya distribution
}

\author{
Edmond Aliaga ${ }^{a}, *$, Shpetim Rexhepi ${ }^{\mathrm{b}}$ \\ a Department of Mathematics, University of Prishtina, Prishtina, Kosovo. \\ ${ }^{b}$ Mother Teresa University, Skopje, North Macedonia.
}

\begin{abstract}
In this paper we introduce a bivariate of q-Durrmeyer variant of generalized Bernstein operators by using Pólya distribution. The convergence rate of these operators is examined by means of the Lipschitz class and the modulus of continuity. Furthermore, we obtain a Voronovskaja type symptotic formula, error estimation in terms of the partial modulus of continuity and Peetre's K-functional.
\end{abstract}

Keywords: Durrmeyer operators, K-functional, modulus of continuity, Pólya distribution.

2020 MSC: 41A36, 40C15, 40G10, 46E30.

(C)2022 All rights reserved.

\section{Introduction}

In the last decade, the application of q-calculus in the approximation theory, mechanics and physics has been one of the main research areas. In approximation theory applications of q-calculus was initiated by Lupaş [12], who first introduced the q-Bernstein polynomials. Another q-based Bernstein operator was introduced in 1997 by Phillips in [15]. Thereafter, many authors studied new classes of q-generalized operators and established many interesting properties. Some important results in this direction are mention in the papers $[1,2,4,5,7,9,11,13,14,16,17]$. Details regarding the definitions and notions of q-calculus can be found at [3].

In 2008, Gupta [8] introduced the q-Durrmeyer operators $D_{n, q}(f)$ and studied some approximation properties of such operators. For $f \in C[0,1], x \in[0,1], n \in \mathbb{N}, 0<q<1$, operators introduced by Gupta [8] are defined as follow:

$$
D_{n_{q}}(f ; x)=[n+1]_{q} \sum_{k=0}^{n} q^{-k} p_{n, k}(q, x) \int_{0}^{1} p_{n, k}(q, q t) f(t) d_{q} t,
$$

where

$$
p_{n, k}(q, x)=\left(\begin{array}{l}
n \\
k
\end{array}\right) x^{k} \prod_{s=0}^{n-k-1}\left(1-q^{s}\right)
$$

\footnotetext{
*Corresponding author

Email addresses: edmond.aliaga@uni-pr.edu (Edmond Aliaga), shpetim.rexhepi@unt.edu.mk (Shpetim Rexhepi)

doi: $10.22436 /$ jmcs.024.03.07
}

Received: 2021-02-01 Revised: 2021-02-17 Accepted: 2021-02-19 
It is proved that for $q=1$, the operators defined by (1.1) reduce to the well known Bernstein-Durrmeyer operators.

Stancu [17], by means of the probabilistic methods, introduced and investigated a linear operators $S_{n}^{(\alpha)}$ which maps the space $C([0,1])$ onto itself and it's defined by

$$
S_{n}^{(\alpha)}(h ; x)=\sum_{k=0}^{n} w_{n, k}(x, \eta) h\left(\frac{k}{n}\right)
$$

where

$$
w_{n, k}\left(z_{1}, \eta\right)=\left[\begin{array}{l}
n \\
k
\end{array}\right] \frac{\prod_{i=0}^{k-1}\left(z_{1}+\eta i\right) \prod_{j=0}^{n-k-1}\left(1-z_{1}+j \eta\right)}{\prod_{s=0}^{n-1}\left(z_{1}+\eta s\right)},
$$

with $\eta$ being a parameter which may depend only on the natural number $n$. If $\eta$ is non-negative, then these operators preserve the positivity of the function $h$.

Nowak [14], introduced the q-variant of the Lupaş operators based on Pólya distribution. For $h \in C(I)$ with $\mathrm{I}=[0,1], \eta>0$, and $0<\mathrm{q}<1$, the operators introduced by Nowak are defined by

$$
\mathrm{L}_{\mathrm{m}_{1}, \mathrm{q}}^{\eta}\left(h ; z_{1}\right)=\sum_{\mathrm{k}_{1}=0}^{\mathrm{m}_{1}} \mathrm{p}_{\mathrm{m}_{1}, \mathrm{k}_{1}}^{\eta}\left(z_{1}\right) \mathrm{h}\left(\frac{\left[k_{1}\right]_{\mathrm{q}}}{\left[\mathrm{m}_{1}\right]_{\mathrm{q}}}\right)
$$

where $p_{m_{1}, k_{1}}^{\eta}\left(z_{1}\right)$ is the Pólya distribution with density function given by

$$
p_{m_{1}, k_{1}}^{\eta}\left(z_{1}\right)=\left[\begin{array}{c}
m_{1} \\
k_{1}
\end{array}\right]_{q} \frac{\prod_{i=0}^{k_{1}-1}\left(z_{1}+\eta[i]_{q}\right) \prod_{j=0}^{m_{1}-k_{1}-1}\left(1-z_{1} q^{j}+\eta[j]_{q}\right)}{\prod_{s=0}^{m_{1}-1}\left(z_{1}+\eta[s]_{q}\right)} .
$$

As special cases, this class contains the following three well-known sequences.

(i) For $\eta=0$, operators defined by (1.2) reduce to the well known q-Bernstein operators, introduced by [14].

(ii) For $q=1$, operators $L_{n, q}^{\eta}\left(h ; z_{1}\right)$ reduce to Bernstein-Stancu operators introduced by [4].

(iii) For $\eta=0$ and $q=1$, operators defined by (1.2) reduce to classical Bernstein operators.

In [10], the q-Durrmayer type modification of operator (1.2) was introduced and studied by Gupta et al. The q-Durrmeyer type operator based on Pólya distribution was defined by

$$
\mathfrak{D}_{\mathrm{m}_{1}, \mathrm{q}_{\mathrm{m}_{1}}}^{\frac{1}{\left[\mathrm{~m}_{1}\right] \mathrm{q}_{1}}}\left(h ; z_{1}\right)=\left[\mathrm{m}_{1}+1\right]_{\mathrm{q}_{1}} \sum_{\mathrm{k}_{1}=0}^{\mathrm{m}_{1}} \mathrm{q}_{1}^{-\mathrm{k}_{1}} \mathrm{p}_{\mathrm{m}_{1}, \mathrm{k}_{1}}^{\frac{1}{\left[\mathrm{~m}_{1}\right] \mathrm{q}_{1}}}\left(z_{1}\right) \int_{0}^{1} p_{\mathrm{m}_{1}, \mathrm{k}_{1}}\left(\mathrm{q}_{1} \mathrm{t}_{1}\right) \mathrm{h}\left(\mathrm{t}_{1}\right) \mathrm{d}_{\mathrm{q}_{1}} \mathrm{t}_{1},
$$

where

$$
p_{m_{1}, k_{1}}^{\frac{1}{\left[m_{1}\right] q_{1}}}(u)=\left[\begin{array}{c}
m_{1} \\
k_{1}
\end{array}\right]_{q_{1}} \frac{\prod_{j=0}^{k_{1}-1}\left(u+\frac{[j]]_{1}}{\left[m_{1}\right]_{q_{1}}}\right) \prod_{j=0}^{m_{1}-k_{1}-1}\left(1-u+\frac{[j]_{q_{1}}}{\left[m_{1}\right]_{q_{1}}}\right)}{\prod_{j=0}^{n_{1}-1}\left(u+\frac{[j]_{q_{1}}}{\left[m_{1}\right]_{q_{1}}}\right)}
$$

and

$$
p_{m_{1}, k_{1}}\left(q_{1} t\right)=\left[\begin{array}{c}
m_{1} \\
k_{1}
\end{array}\right]_{q_{1}} q_{1}^{k_{1}} t^{k_{1}} \prod_{j=0}^{m_{1}-k_{1}-1}\left(1-q_{1}^{j+1} t\right) .
$$

In order to prove Lemmas 1.2 and 2.1, we will establish the following statements for operators defined by (1.3). 
Lemma 1.1. For each positive numbers $C_{s, r}\left(q_{1}\right)>0, s=\{0,1, \ldots, r\}, r \in \mathbb{N}$ the following statements holds

$$
\mathfrak{D}_{\mathrm{m}_{1}, \mathrm{q}_{1}}^{\frac{1}{\left[\mathrm{~m}_{1}\right]}}\left(e_{\mathrm{r}} ; z_{1}\right)=\frac{\left[\mathrm{m}_{1}+1\right]_{\mathrm{q}_{1}} !}{\left[\mathrm{m}_{1}+\mathrm{r}+1\right]_{\mathrm{q}_{1}} !} \sum_{\mathrm{k}_{1}=0}^{\mathrm{r}} l_{\mathfrak{i}}(\mathrm{r})\left[\mathrm{m}_{1}\right]_{\mathrm{q}_{1}}^{k_{1}} \mathrm{~L}_{\mathrm{m}_{1}, \mathrm{q}_{1}}^{1 /\left[\mathrm{m}_{1}\right]_{\mathrm{q}_{1}}}\left(e_{k_{1}} ; z_{1}\right),
$$

where $\mathrm{e}_{\mathrm{r}}\left(z_{1}\right)=z_{1}^{\mathrm{r}}$ and $\sum_{\mathrm{k}_{1}=0}^{\mathrm{r}} \mathrm{l}_{\mathrm{i}}(\mathrm{r})\left[\mathrm{m}_{1}\right]_{\mathbf{q}_{1}}^{\mathrm{k}_{1}}=\left[\mathrm{m}_{1}+1\right]_{\mathbf{q}_{1}}\left[\mathrm{~m}_{1}+2\right]_{\mathbf{q}_{1}} \ldots\left[\mathrm{m}_{1}+\mathrm{r}\right]_{\mathbf{q}_{1}}$.

Proof. Indeed, we have

$$
\begin{aligned}
\mathfrak{D}_{m_{1}, q_{1}}^{\frac{1}{\left.m_{1}\right] q_{1}}}\left(e_{r} ; z_{1}\right) & =\left[m_{1}+1\right]_{q_{1}} \sum_{k_{1}=0}^{m_{1}} q_{1}^{-k_{1}} p_{m_{1}, k_{1}}^{\frac{1}{\left.m_{1}\right] q_{1}}}\left(z_{1}\right) \int_{0}^{1} p_{m_{1}, k_{1}}\left(q_{1} t_{1}\right) e_{r}\left(t_{1}\right) d_{q_{1}} t_{1} \\
& =\left[m_{1}+1\right]_{q_{1}} \sum_{k_{1}=0}^{m_{1}} q_{1}^{-k_{1}} p_{m_{1}, k_{1}}^{\frac{1}{\left[m_{1}\right] q_{1}}}\left(z_{1}\right) q_{1}^{k_{1}} \frac{\left.\left[m_{1}\right]_{q_{1}} !\left[k_{1}+r\right]\right]_{q_{1}} !}{\left[k_{1}\right]_{q_{1}} !\left[m_{1}+r+1\right]_{q_{1}} !} .
\end{aligned}
$$

Using $\left[s+k_{1}\right]_{q_{1}}=[s]_{q_{1}}+q_{1}^{s}\left[k_{1}\right]_{q_{1}}$, we obtain

$$
\frac{\left[k_{1}+r\right]_{\mathbf{q}_{1}} !}{\left[k_{1}\right]_{\mathbf{q}_{1}} !}=\left[k_{1}+1\right]_{\mathbf{q}_{1}}\left[k_{1}+2\right]_{\mathbf{q}_{1}} \cdots\left[k_{1}+r\right]_{\mathbf{q}_{1}}=\prod_{s=1}^{r}\left([s]_{\mathbf{q}_{1}}+\mathbf{q}_{1}^{s}\left[k_{1}\right]_{\mathbf{q}_{1}}\right)=\sum_{s=1}^{r} c_{s, r}\left(q_{1}\right)\left[k_{1}\right]_{\mathbf{q}_{1}}^{s}
$$

where $C_{s, r}\left(q_{1}\right), s=1,2, \ldots, r$ are the constants independent of $k_{1}$. Hence

$$
\begin{aligned}
\mathfrak{D}_{m_{1}, q_{1}}^{\frac{1}{\left[m_{1}\right] q_{1}}}\left(e_{r} ; z_{1}\right) & =\frac{\left[m_{1}+1\right]_{\mathfrak{q}_{1}} !}{\left[m_{1}+r+1\right]_{\mathfrak{q}_{1}} !} \sum_{k_{1}=0}^{m_{1}} p_{m_{1}, k_{1}}^{[m 1]} \frac{1}{\left[m 1 q_{1}\right.}\left(z_{1}\right) \sum_{s=1}^{r} C_{s, r}\left(q_{m_{1}}\right)\left[k_{1}\right]_{q_{1}}^{s} \\
& =\frac{\left[m_{1}+1\right]_{q_{1}} !}{\left[m_{1}+r+1\right]_{q_{1}} !} \sum_{s=1}^{r} C_{s, r}\left(q_{1}\right)\left[m_{1}\right]_{q_{1}}^{s} L_{m_{1}, q_{1}}^{1 /\left[m_{1}\right]_{q_{1}}}\left(e_{s} ; z_{1}\right) .
\end{aligned}
$$

Lemma 1.2 ([10]). The q-Durrmayer operators defined by (1.3) satisfy the equalities
i) $\mathfrak{D}_{\mathrm{m}_{1}, \mathbf{q}_{1}}^{\frac{1}{\left[\mathrm{~m}_{1}\right] \mathrm{q}_{1}}}\left(e_{0} ; z_{1}\right)=1$;
ii) $\mathfrak{D}_{\mathfrak{m}_{1}, \mathfrak{q}_{1}}^{\frac{1}{\left.\mathrm{~m}_{1}\right] \mathrm{q}_{1}}}\left(e_{1} ; z_{1}\right)=\frac{\mathfrak{q}_{1}\left[\mathrm{~m}_{1}\right]_{\mathfrak{q}_{1}} z_{1}+1}{\left.\left[\mathrm{~m}_{1}+2\right]\right]_{\mathfrak{q}_{1}}}$;

iii)

$$
\begin{aligned}
& \mathfrak{D}_{\mathrm{m}_{1}, \mathrm{~m}_{2}, \mathbf{q}_{1}, \mathbf{q}_{2}}^{\frac{1}{\left[\mathrm{~m}_{2}\right] \mathbf{q}_{1}}}\left(\frac{1}{\left.m_{2}\right]}\left(e_{20} ; z_{1}, z_{2}\right)=\frac{\left[m_{1}\right]_{\mathbf{q}_{1}}^{3} q_{1}^{3}}{\left(1+\left[m_{1}\right]_{\mathbf{q}_{1}}\right)\left[m_{1}+2\right]_{\mathbf{q}_{1}}\left[m_{1}+3\right]_{\mathbf{q}_{1}}}\left(z_{1}\left(z_{1}+\frac{1}{\left[m_{1}\right]_{\mathbf{q}_{1}}}\right)+\frac{z_{1}\left(1-z_{1}\right)}{\left[m_{1}\right]_{\mathbf{q}_{1}}}\right)\right. \\
& +\frac{\mathrm{q}_{1}\left(2 \mathrm{q}_{1}+1\right)\left[\mathrm{m}_{1}\right]_{\mathrm{q}_{1}} z_{1}}{\left[\mathrm{~m}_{1}+2\right]_{\mathrm{q}_{1}}\left[\mathrm{~m}_{1}+3\right]_{\mathrm{q}_{1}}}+\frac{\mathrm{q}_{1}+1}{\left[\mathrm{~m}_{1}+2\right]_{\mathrm{q}_{1}}\left[\mathrm{~m}_{1}+3\right]_{\mathrm{q}_{1}}} ;
\end{aligned}
$$

iv)

$$
\begin{aligned}
& \mathfrak{D}_{\mathrm{m}_{1}, \mathrm{q}_{1}}^{\frac{1}{\left[\mathrm{~m}_{1}\right]}}\left(e_{30} ; z_{1}, z_{2}\right)=\frac{\mathrm{q}_{1}^{5}\left[\mathrm{~m}_{1}\right]_{\mathrm{q}_{1}}^{3}}{\prod_{i=0}^{2}\left[\mathrm{~m}_{1}+i+2\right]_{\mathrm{q}_{1}}} \frac{1}{\prod_{i=0}^{2}\left(1+[i]_{\mathbf{q}_{1}} /\left[\mathrm{m}_{1}\right]_{\mathrm{q}_{1}}\right)} \sum_{k=0}^{2} \frac{A_{k}\left(\mathrm{q}_{1}, 1 /\left[\mathrm{m}_{1}\right]_{\mathrm{q}_{1}} ; z_{1}\right)}{\left[\mathrm{m}_{1}\right]_{\mathbf{q}_{1}}^{\mathrm{k}}} \\
& \left.+\frac{\left(\mathrm{q}_{1}^{3}+3 \mathrm{q}_{1}^{4}+2 \mathrm{q}_{1}^{5}\right)\left[\mathrm{m}_{1}\right]_{\mathbf{q}_{1}}^{3}}{\left(1+\left[\mathrm{m}_{1}\right]_{\mathbf{q}_{1}}\right) \prod_{i=0}^{2}\left[\mathrm{~m}_{1}+i+2\right]_{\mathbf{q}_{1}}}\left(z_{1}\left(z_{1}+1 /\left[\mathrm{m}_{1}\right]_{\mathbf{q}_{1}}\right)+z_{1}\left(1-z_{1}\right) /\left[\mathrm{m}_{1}\right]_{\mathbf{q}_{1}}\right)\right) \\
& +\frac{\left(\mathrm{q}_{1}+3 \mathrm{q}_{1}^{2}+4 \mathrm{q}_{1}^{3}+3 \mathrm{q}_{1}^{4}\right)\left[\mathrm{m}_{1}\right]_{\mathrm{q}_{1}} z_{1}}{\prod_{i=0}^{2}\left[\mathrm{~m}_{1}+i+2\right]_{\mathrm{q}_{1}}}+\frac{1+2 \mathrm{q}_{1}+2 \mathrm{q}_{1}^{2}+2 \mathrm{q}_{1}^{3}}{\prod_{i=0}^{2}\left[\mathrm{~m}_{1}+i+2\right]_{\mathrm{q}_{1}}}
\end{aligned}
$$


v)

$$
\begin{aligned}
& \mathfrak{D}_{\mathrm{m}_{1}, \mathrm{q}_{1}}^{\frac{1}{\left[\mathrm{~m}_{1}\right]}}\left(e_{40} ; z_{1}, z_{2}\right) \\
& =\frac{\mathbf{q}_{1}^{9}\left[m_{1}\right]_{\mathbf{q}_{1}}^{4}}{\prod_{i=0}^{3}\left[m_{1}+i+2\right]_{\mathbf{q}_{1}}} \frac{1}{\prod_{i=0}^{3}\left(1+[i]_{\mathbf{q}_{1}} /\left[m_{1}\right]_{\mathbf{q}_{1}}\right)} \sum_{k=0}^{3} \frac{A_{k}^{*}\left(\mathbf{q}_{1}, 1 /\left[m_{1}\right]_{\mathbf{q}_{1}} ; z_{1}\right)}{\left[m_{1}\right]_{\mathbf{q}_{1}}^{k}} \\
& +\frac{\left(q_{1}^{5}+q_{1}^{6}+2 q_{1}^{7}+4 q_{1}^{8}+2 q_{1}^{9}\right)\left[m_{1}\right]_{q_{1}}^{3}}{\prod_{i=0}^{3}\left[m_{1}+i+2\right]_{q_{1}}} \frac{1}{\prod_{i=0}^{2}\left(1+[i]_{q_{1}} /\left[m_{1}\right]_{q_{1}}\right)} \sum_{k=0}^{2} \frac{A_{k}\left(q_{1}, 1 /\left[m_{1}\right]_{q_{1}} ; z_{1}\right)}{\left[m_{1}\right]_{q_{1}}^{k}} \\
& \left.+\frac{\left(q_{1}^{3}+4 q_{1}^{4}+7 q_{1}^{5}+9 q_{1}^{6}+9 q_{1}^{7}+5 q_{1}^{8}\right)\left[m_{1}\right]_{\mathbf{q}_{1}}^{3}}{\left(1+\left[m_{1}\right]_{q_{1}}\right) \prod_{i=0}^{3}\left[m_{1}+i+2\right]_{q_{1}}}\left(z_{1}\left(z_{1}+1 /\left[m_{1}\right]_{q_{1}}\right)+z_{1}\left(1-z_{1}\right) /\left[m_{1}\right]_{q_{1}}\right)\right) \\
& +\frac{\left(q_{1}+4 q_{1}^{2}+8 q_{1}^{3}+12 q_{1}^{4}+12 q_{1}^{5}+9 q_{1}^{6}+5 q_{1}^{7}\right)\left[m_{1}\right]_{q_{1}} z_{1}}{\prod_{i=0}^{3}\left[m_{1}+i+2\right]_{q_{1}}}+\frac{\left(1+3 q_{1}+5 q_{1}^{2}+7 q_{1}^{3}+6 q_{1}^{4}+4 q_{1}^{5}+2 q_{1}^{6}\right)}{\prod_{i=0}^{3}\left[m_{1}+i+2\right]_{q_{1}}} ;
\end{aligned}
$$

where

$$
\begin{aligned}
& A_{0}\left(\mathrm{q}_{1} ; 1 /\left[\mathrm{m}_{1}\right]_{\mathbf{q}_{1}} ; z_{1}\right)=z_{1}\left(z_{1}+1 /\left[\mathrm{m}_{1}\right]_{\mathbf{q}_{1}}\right)\left(z_{1}+[2]_{\mathbf{q}_{1}} /\left[\mathrm{m}_{1}\right]_{\mathbf{q}_{1}}\right), \\
& \mathrm{A}_{1}\left(\mathrm{q}_{1} ; 1 /\left[\mathrm{m}_{1}\right]_{\mathbf{q}_{1}} ; z_{1}\right)=z_{1}\left(1-z_{1}\right)\left(z_{1}+1 /\left[\mathrm{m}_{1}\right]_{\mathbf{q}_{1}}\right)\left(2+\mathrm{q}_{1}\right) \text {, } \\
& A_{2}\left(\mathrm{q}_{1} ; 1 /\left[\mathrm{m}_{1}\right]_{\mathrm{q}_{1}} ; z_{1}\right)=z_{1}\left(1-z_{1}\right)\left(1-[2]_{\mathbf{q}_{1}} z_{1}\right) \text {, } \\
& A_{0}^{*}\left(\mathrm{q}_{1} ; 1 /\left[\mathrm{m}_{1}\right]_{\mathbf{q}_{1}} ; z_{1}\right)=z_{1}\left(z_{1}+1 /\left[\mathrm{m}_{1}\right]_{\mathbf{q}_{1}}\right)\left(z_{1}+[2]_{\mathbf{q}_{1}} /\left[\mathrm{m}_{1}\right]_{\mathbf{q}_{1}}\right)\left(z_{1}+[3]_{\mathbf{q}_{1}} /\left[\mathrm{m}_{1}\right]_{\mathbf{q}_{1}}\right), \\
& A_{1}^{*}\left(\mathrm{q}_{1} ; 1 /\left[\mathrm{m}_{1}\right]_{\mathrm{q}_{1}} ; z_{1}\right)=z_{1}\left(1-z_{1}\right)\left(z_{1}+1 /\left[\mathrm{m}_{1}\right]_{\mathbf{q}_{1}}\right)\left(z_{1}+[2]_{\mathbf{q}_{1}} /\left[\mathrm{m}_{1}\right]_{\mathbf{q}_{1}}\right)\left(\mathrm{q}_{1}^{2}+2 \mathrm{q}_{1}+3\right) \text {, } \\
& A_{2}^{*}\left(\mathrm{q}_{1} ; 1 /\left[\mathrm{m}_{1}\right]_{\mathbf{q}_{1}} ; z_{1}\right)=z_{1}\left(1-z_{1}\right)\left(z_{1}+1 /\left[\mathrm{m}_{1}\right]_{\mathbf{q}_{1}}\right)\left(\left(\mathrm{q}_{1}^{2}+3 \mathrm{q}_{1}+3\right) z_{1}\left(z_{1}+1 /\left[\mathrm{m}_{1}\right]_{\mathbf{q}_{1}}\right)\right. \\
& \left.-[2]_{\mathbf{q}_{1}}^{2}\left(z_{1}+1 /\left[\mathrm{m}_{1}\right]_{\mathbf{q}_{1}}-[2]_{\mathbf{q}_{1}} z_{1}\left(1+[3]_{\mathbf{q}_{1}} /\left[\mathrm{m}_{1}\right]_{\mathbf{q}_{1}}\right)\right)\right), \\
& A_{3}^{*}\left(\mathrm{q}_{1} ; 1 /\left[\mathrm{m}_{1}\right]_{\mathbf{q}_{1}} ; z_{1}\right)=z_{1}\left(1-z_{1}\right)\left(z_{1}+1 /\left[\mathrm{m}_{1}\right]_{\mathbf{q}_{1}}\right)\left([2]_{\mathbf{q}_{1}} z_{1}\left([3]_{\mathbf{q}_{1}} z_{1}-\mathrm{q}_{1}-2\right)+1-\mathrm{q}_{1} /\left[\mathrm{m}_{1}\right]_{\mathbf{q}_{1}}\right) .
\end{aligned}
$$

The aim of paper is to study properties of these operators, the study of the uniform convergence, as well as rate of convergence in terms of the Lipschitz class function. Furthermore, we obtain a Voronovskaja type symptotic formula, error estimation in terms of modulus of continuity and Petree K-functional.

\section{Construction of the Bivariate q-Durrmeyer-Pólya operators}

In what follows, let $\mathrm{I}=[0,1], \mathrm{I}^{2}=\mathrm{I} \times \mathrm{I}$ and $\left\{\mathrm{q}_{\mathrm{m}_{\mathrm{i}}}\right\}$ be the sequences of real numbers such that $0<\mathrm{q}_{\mathrm{m}_{\mathrm{i}}}<$ 1, $\lim _{m_{i} \rightarrow \infty} q_{m_{i}}=1$ and $\lim _{m_{i} \rightarrow \infty} q_{m_{i}}^{m_{i}}=a, a \in[0,1), i=1$, Further, let $\delta_{m_{1}}\left(z_{1}\right)=\mathfrak{D}_{m_{1}, q_{m_{1}}}^{\frac{1}{m_{1} 1 q_{m_{1}}}}\left(\left(u-z_{1}\right)^{2}, q_{m_{1}}, z_{1}\right)$ and $\left.\delta_{m_{2}}\left(z_{2}\right)=\mathfrak{D}_{m_{2}, q_{m_{2}}}^{\frac{1}{\left[m_{2}\right] m_{2}}}\left(v-z_{2}\right)^{2}, q_{m_{2}}, z_{2}\right)$. Let $\mathfrak{D}_{m_{i}, q_{m_{i}}}^{\frac{1}{\left[m_{i} q_{m_{i}}\right.}}: C(I) \rightarrow C(I), i=1,2$ defined for each positive integers $m_{1}, m_{2}$ and $0<q_{m_{1}}, q_{m_{2}}<1$, and any $h \in C(I), g \in C(I)$, respectively by

$$
\begin{aligned}
& \mathfrak{D}_{m_{1}, q_{m_{1}}}^{\frac{1}{\left[m_{1} q_{m_{1}}\right.}}\left(h ; z_{1}\right)=\left[m_{1}+1\right]_{q_{m_{1}}} \sum_{k_{1}=0}^{m_{1}} q_{m_{1}}^{-k_{1}} p_{m_{1}, k_{1}}^{\frac{1}{\left[m_{1}\right] q_{m_{1}}}}\left(z_{1}\right) \int_{0}^{1} p_{m_{1}, k_{1}}\left(q_{m_{1}} t_{1}\right) h\left(t_{1}\right) d_{q_{m_{1}}} t_{1}, \\
& \mathfrak{D}_{m_{2}, q_{m_{2}}}^{\left[\frac{1}{\left[m_{2}\right] q_{2}}\right.}\left(h ; z_{2}\right)=\left[m_{2}+1\right]_{q_{m_{2}}} \sum_{k_{2}=0}^{m_{2}} q_{m_{2}}^{-k_{2}} p_{m_{2}, k_{2}}^{\left[m_{2}\right] q_{m_{2}}}\left(z_{2}\right) \int_{0}^{1} p_{m_{2}, k_{2}}\left(q_{m_{2}} t_{2}\right) h\left(t_{2}\right) d_{q_{m_{2}}} t_{2} .
\end{aligned}
$$


The parametric extension of (2.1) and (2.2) are the operators $\mathfrak{D}_{\mathrm{m}_{1}, \mathrm{q}_{\mathrm{m}_{1}}}^{\frac{1}{\left.\mathrm{~m}_{1}\right] \mathrm{q}_{1}}}, \mathfrak{D}_{\mathrm{m}_{2}, \mathrm{q}_{\mathrm{m}_{2}}}^{\frac{1}{\left.\mathrm{~m}_{2}\right] \mathrm{m}_{2}}}: \mathrm{C}\left(\mathrm{I}^{2}\right) \rightarrow \mathrm{C}\left(\mathrm{I}^{2}\right)$, defined for each positive integers $m_{1}, m_{2}$ and $h \in C\left(I^{2}\right)$, as follows:

$$
\begin{aligned}
& \mathfrak{D}_{\mathrm{m}_{1}, \mathbf{q}_{\mathrm{m}_{1}}}^{z_{1, \frac{1}{\left.m_{1}\right] \mathrm{m}_{1}}}}\left(h ; z_{1}\right)=\left[\mathrm{m}_{1}+1\right]_{\mathbf{q}_{\mathrm{m}_{1}}} \sum_{\mathrm{k}_{1}=0}^{\mathrm{m}_{1}} \mathbf{q}_{\mathrm{m}_{1}}^{-\mathrm{k}_{1}} \mathrm{p}_{\mathrm{m}_{1}, \mathrm{k}_{1}}^{\frac{1}{\left[\mathrm{~m}_{1}\right] \mathrm{m}_{1}}}\left(z_{1}\right) \int_{0}^{1} p_{\mathrm{m}_{1}, \mathrm{k}_{1}}\left(\mathbf{q}_{\mathrm{m}_{1}} \mathrm{t}_{1}\right) \mathrm{h}\left(\mathrm{t}_{1}, v\right) \mathrm{d}_{\mathrm{q}_{\mathrm{m}_{1}}} \mathrm{t}_{1}, \\
& \mathfrak{D}_{m_{2}, \mathbf{q}_{m_{2}}}^{z_{2, \frac{1}{\left.m_{2}\right] m_{2}}}}\left(h ; z_{2}\right)=\left[m_{2}+1\right]_{\mathfrak{q}_{m_{2}}} \sum_{k_{2}=0}^{m_{2}} q_{m_{2}}^{-k_{2}} p_{m_{2}, k_{2}}^{\frac{1}{\left\lfloor m_{2}\right\rfloor m_{2}}}\left(z_{2}\right) \int_{0}^{1} p_{m_{2}, k_{2}}\left(q_{m_{2}} t_{2}\right) h\left(u, t_{2}\right) d_{q_{m_{2}}} t_{2} .
\end{aligned}
$$

Motivated by Gupta et al. [10], for $h \in C\left(I^{2}\right)$ and $0<q_{m_{1}}, q_{m_{2}}<1$, we construct the bivariate extension of the univariate q-Durrmeyer operators based Pólya distribution for each positive integers $\mathrm{m}_{1}, \mathrm{~m}_{2}$ as follows:

$$
\begin{aligned}
& \mathfrak{D}_{m_{1}, m_{2}, q_{m_{1}}, q_{m_{2}}}^{\frac{1}{\left[m_{1}\right] m_{m_{1}}}} \frac{1}{\left[m_{1}\right] q_{m_{2}}}\left(h ; z_{1}, z_{2}\right)=\left[m_{1}+1\right]_{\mathbf{q}_{m_{1}}}\left[m_{2}+1\right]_{q_{m_{2}}} \sum_{k_{1}=0}^{m_{1}} \sum_{k_{2}=0}^{m_{2}} q_{m_{1}}^{-k_{1}} q_{m_{2}}^{-k_{2}} p_{m_{1}, k_{1}}^{\frac{1}{\left[m_{1}\right] m_{1}}}\left(z_{1}\right) p_{m_{2}, k_{2}}^{\frac{1}{\left[m_{2}\right] m_{2}}}\left(z_{2}\right) \\
& \cdot \int_{0}^{1} \int_{0}^{1} p_{m_{1}, k_{1}}\left(q_{m_{1}} t_{1}\right) p_{m_{2}, k_{2}}\left(q_{m_{2}} t_{2}\right) h\left(t_{1}, t_{2}\right) d_{q_{m_{1}}} t_{1} d_{q_{m_{2}}} t_{2}
\end{aligned}
$$

In what follows, let $e_{i j}: I^{2} \rightarrow R, e_{i j}\left(z_{1}, z_{2}\right)=z_{1}^{i} z_{2}^{j},\left(z_{1}, z_{2}\right) \in I^{2},(i, j) \in N^{0} \times N^{0}$ with $i+j \leqslant 4$ be the two dimensional test function. Further, we give some lemmas.

Lemma 2.1. The bivariate q-Durrmayer operators based on Pólya distribution defined by (2.3), satisfy the equalities
i) $\mathfrak{D}_{\mathrm{m}_{1}, \mathrm{~m}_{2}, \mathrm{q}_{\mathrm{m}_{1}}, \mathrm{q}_{\mathrm{m}_{2}}}^{\frac{1}{\left[\mathrm{~m}_{1}\right] \mathrm{q}_{\mathrm{m}_{1}}} \frac{1}{\left[\mathrm{~m}_{\mathrm{m}_{2}}\right.}}\left(e_{00} ; z_{1}, z_{2}\right)=1$;

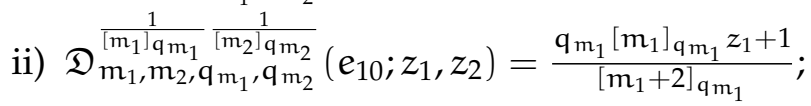

iii)

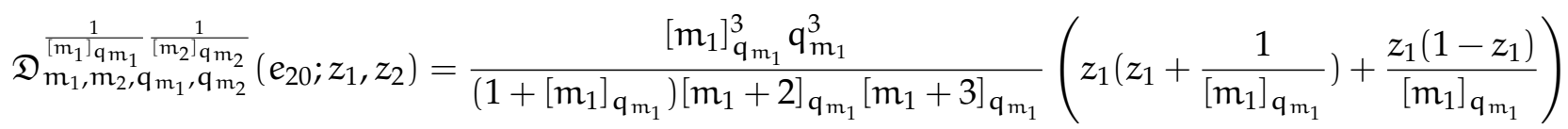

$$
\begin{aligned}
& +\frac{\mathbf{q}_{m_{1}}\left(2 q_{m_{1}}+1\right)\left[m_{1}\right]_{\mathfrak{q}_{m_{1}}} z_{1}}{\left[m_{1}+2\right]_{\mathbf{q}_{m_{1}}}\left[m_{1}+3\right]_{\mathfrak{q}_{m_{1}}}}+\frac{\mathbf{q}_{m_{1}}+1}{\left[m_{1}+2\right]_{\mathfrak{q}_{m_{1}}}\left[m_{1}+3\right]_{\mathfrak{q}_{m_{1}}}} \text {; }
\end{aligned}
$$

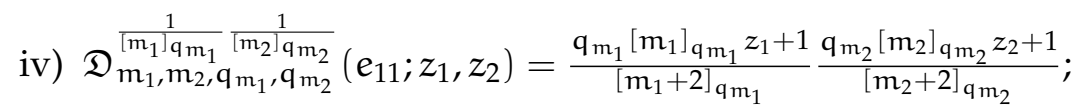

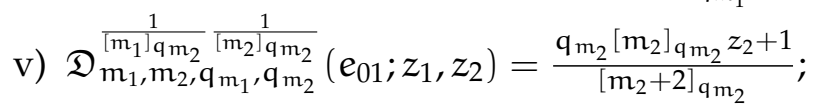

vi)

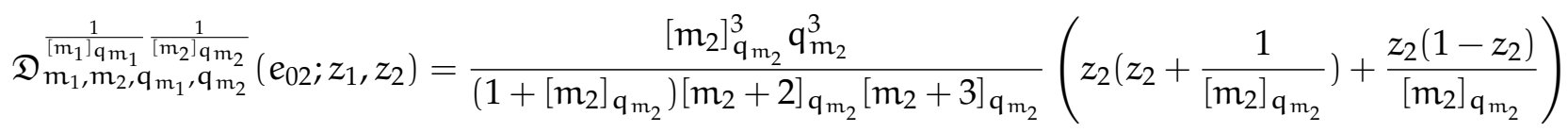

$$
\begin{aligned}
& +\frac{\mathbf{q}_{m_{2}}\left(2 q_{m_{2}}+1\right)\left[m_{2}\right]_{\mathbf{q}_{m_{2}}} z_{2}}{\left[m_{2}+2\right]_{\mathbf{q}_{m_{2}}}\left[m_{2}+3\right]_{\mathbf{q}_{m_{2}}}}+\frac{\mathbf{q}_{m_{2}}+1}{\left[m_{2}+2\right]_{\mathbf{q}_{m_{2}}}\left[m_{2}+3\right]_{\mathbf{q}_{m_{2}}}} \text {; }
\end{aligned}
$$

vii)

$$
\left.\mathfrak{D}_{m_{1}, m_{2}, q_{m_{1}}, q_{m_{2}}}^{\frac{1}{\left[m_{1}\right] m_{m_{1}}}} \frac{1}{\left[m_{2}\right] \mathbf{q}_{2}} ; z_{1}, z_{2}\right)
$$




$$
\begin{aligned}
& =\frac{\mathrm{q}_{\mathrm{m}_{1}}^{5}\left[\mathrm{~m}_{1}\right]_{\mathbf{q}_{\mathrm{m}_{1}}}^{3}}{\prod_{i=0}^{2}\left[\mathrm{~m}_{1}+i+2\right]_{\mathbf{q}_{\mathrm{m}_{1}}}} \frac{1}{\prod_{i=0}^{2}\left(1+[i]_{\mathbf{q}_{\mathrm{m}_{1}}} /\left[\mathrm{m}_{1}\right]_{\mathrm{q}_{\mathrm{m}_{1}}}\right)} \sum_{k=0}^{2} \frac{A_{k}\left(\mathrm{q}_{\mathrm{m}_{1}, 1},\left[\mathrm{~m}_{1}\right]_{\left.\mathbf{q}_{\mathrm{m}_{1}} ; z_{1}\right)^{k}}\right.}{\left[\mathrm{m}_{1}\right]_{\mathrm{q}_{\mathrm{m}_{1}}}} \\
& \left.+\frac{\left(\mathbf{q}_{\mathrm{m}_{1}}^{3}+3 \mathbf{q}_{\mathrm{m}_{1}}^{4}+2 \mathbf{q}_{\mathrm{m}_{1}}^{5}\right)\left[\mathrm{m}_{1}\right]_{\mathbf{q}_{\mathrm{m}_{1}}}^{3}}{\left(1+\left[\mathrm{m}_{1}\right]_{\mathbf{q}_{\mathrm{m}_{1}}}\right) \prod_{i=0}^{2}\left[\mathrm{~m}_{1}+i+2\right]_{\mathbf{q}_{\mathrm{m}_{1}}}}\left(z_{1}\left(z_{1}+1 /\left[\mathrm{m}_{1}\right]_{\mathbf{q}_{\mathrm{m}_{1}}}\right)+z_{1}\left(1-z_{1}\right) /\left[\mathrm{m}_{1}\right]_{\mathbf{q}_{\mathrm{m}_{1}}}\right)\right) \\
& +\frac{\left(q_{m_{1}}+3 q_{m_{1}}^{2}+4 q_{m_{1}}^{3}+3 q_{m_{1}}^{4}\right)\left[m_{1}\right]_{q_{m_{1}}} z_{1}}{\prod_{i=0}^{2}\left[m_{1}+i+2\right]_{q_{m_{1}}}}+\frac{1+2 q_{m_{1}}+2 q_{m_{1}}^{2}+2 q_{m_{1}}^{3}}{\prod_{i=0}^{2}\left[m_{1}+i+2\right]_{q_{m_{1}}}}
\end{aligned}
$$

viii)

$$
\begin{aligned}
& \mathfrak{D}_{\mathrm{m}_{1}, \mathrm{~m}_{2}, \mathbf{q}_{\mathrm{m}_{1}}, \mathbf{q}_{\mathrm{m}_{2}}}^{\frac{1}{\left[\mathrm{~m}_{1}\right] \mathrm{q}_{\mathrm{m}_{2}}} \frac{1}{\left[\mathrm{~m}_{2}\right.}}\left(\boldsymbol{e}_{40} ; z_{1}, z_{2}\right) \\
& =\frac{\mathbf{q}_{m_{1}}^{9}\left[m_{1}\right]_{\mathbf{q}_{\mathfrak{m}_{1}}}^{4}}{\prod_{i=0}^{3}\left[m_{1}+i+2\right]_{\mathbf{q}_{\mathrm{m}_{1}}}} \frac{1}{\prod_{i=0}^{3}\left(1+[i]_{\mathbf{q}_{\mathrm{m}_{1}}} /\left[\mathrm{m}_{1}\right]_{\mathbf{q}_{\mathrm{m}_{1}}}\right)} \sum_{k=0}^{3} \frac{A_{k}^{*}\left(\mathbf{q}_{\mathrm{m}_{1}}, 1 /\left[\mathrm{m}_{1}\right]_{\mathbf{q}_{\mathrm{m}_{1}}} ; z_{1}\right)}{\left[\mathrm{m}_{1}\right]_{\mathbf{q}_{\mathrm{m}_{1}}}^{k}} \\
& +\frac{\left(\mathbf{q}_{\mathrm{m}_{1}}^{5}+\mathbf{q}_{\mathrm{m}_{1}}^{6}+2 \mathbf{q}_{\mathrm{m}_{1}}^{7}+4 \mathbf{q}_{\mathrm{m}_{1}}^{8}+2 \mathbf{q}_{\mathrm{m}_{1}}^{9}\right)\left[\mathrm{m}_{1}\right]_{\mathbf{q}_{\mathrm{m}_{1}}}^{3}}{\prod_{i=0}^{3}\left[\mathrm{~m}_{1}+i+2\right]_{\mathbf{q}_{\mathrm{m}_{1}}}} \frac{1}{\prod_{i=0}^{2}\left(1+[i]_{\left.\mathbf{q}_{\mathrm{m}_{1}} /\left[\mathrm{m}_{1}\right]_{\mathbf{q}_{\mathrm{m}_{1}}}\right)}\right.} \sum_{k=0}^{2} \frac{A_{k}\left(\mathbf{q}_{\mathrm{m}_{1}}, 1 /\left[\mathrm{m}_{1}\right]_{\mathbf{q}_{\mathrm{m}_{1}} ; z_{1}}\right)}{\left[\mathrm{m}_{1}\right]_{\mathbf{q}_{\mathrm{m}_{1}}}^{\mathrm{k}}} \\
& \left.+\frac{\left(\mathbf{q}_{\mathrm{m}_{1}}^{3}+4 \mathbf{q}_{\mathrm{m}_{1}}^{4}+7 \mathbf{q}_{\mathrm{m}_{1}}^{5}+9 \mathbf{q}_{\mathrm{m}_{1}}^{6}+9 \mathbf{q}_{\mathrm{m}_{1}}^{7}+5 \mathbf{q}_{\mathrm{m}_{1}}^{8}\right)\left[\mathrm{m}_{1}\right]_{\mathbf{q}_{\mathrm{m}_{1}}}^{3}}{\left(1+\left[\mathrm{m}_{1}\right]_{\mathbf{q}_{\mathrm{m}_{1}}}\right) \prod_{i=0}^{3}\left[\mathrm{~m}_{1}+i+2\right]_{\mathbf{q}_{\mathrm{m}_{1}}}}\left(z_{1}\left(z_{1}+1 /\left[\mathrm{m}_{1}\right]_{\mathbf{q}_{\mathrm{m}_{1}}}\right)+z_{1}\left(1-z_{1}\right) /\left[\mathrm{m}_{1}\right]_{\mathbf{q}_{\mathrm{m}_{1}}}\right)\right) \\
& +\frac{\left(q_{m_{1}}+4 q_{m_{1}}^{2}+8 q_{m_{1}}^{3}+12 q_{m_{1}}^{4}+12 q_{m_{1}}^{5}+9 q_{m_{1}}^{6}+5 q_{m_{1}}^{7}\right)\left[m_{1}\right]_{q_{m_{1}}} z_{1}}{\prod_{i=0}^{3}\left[m_{1}+i+2\right]_{q_{m_{1}}}} \\
& +\frac{\left(1+3 q_{m_{1}}+5 q_{m_{1}}^{2}+7 q_{m_{1}}^{3}+6 q_{m_{1}}^{4}+4 q_{m_{1}}^{5}+2 q_{m_{1}}^{6}\right)}{\prod_{i=0}^{3}\left[m_{1}+i+2\right]_{q_{m_{1}}}}
\end{aligned}
$$

where $A_{i}\left(q_{m_{1}} ; 1 /\left[m_{1}\right]_{\mathbf{q}_{m_{1}}} ; z_{1}\right)$ and $A_{k}^{*}\left(q_{m_{1}}, 1 /\left[m_{1}\right]_{\mathfrak{q}_{m_{1}}} ; z_{1}\right) i=1,2,3$ are defined in Lemma 1.2.

Proof. This lemma follows easily while applying previous lemmas, as well as taking into account the definition of q-Durrmeyer-Pólya operators (2.3), and obtains the following indentities:

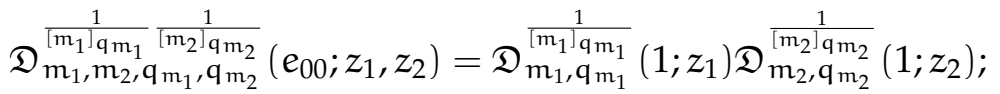

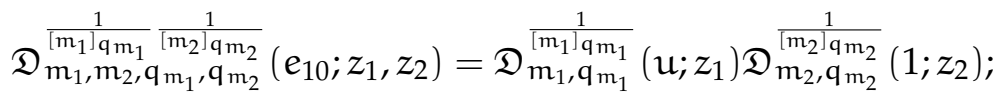

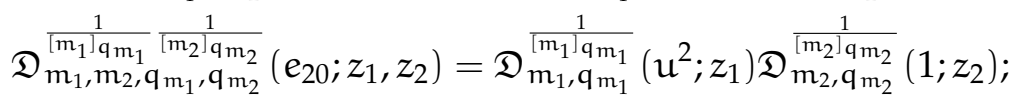

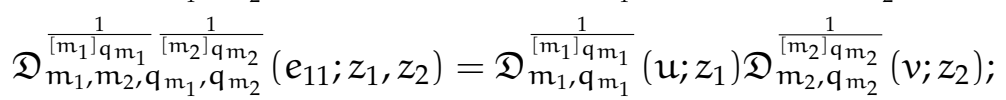

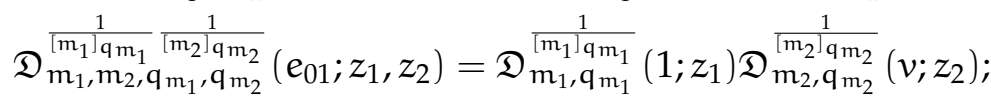

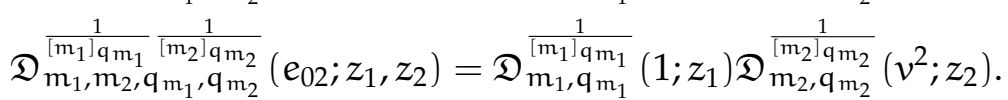


Lemma 2.2. For operators defined by (2.3), the following identities hold true

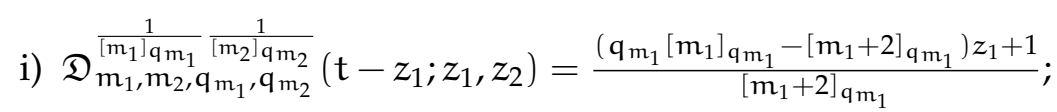

ii)

$$
\begin{aligned}
& \mathfrak{D}_{\mathrm{m}_{1}, \mathrm{~m}_{2}, \mathbf{q}_{\mathrm{m}_{1}}, \mathbf{q}_{\mathrm{m}_{2}}}^{\frac{1}{\left[m_{2}\right] \mathrm{m}_{2}}}\left(\left(\mathrm{t}-z_{1}\right)^{2} ; z_{1}, z_{2}\right) \\
& =\left(1+\frac{\left(\left[m_{1}\right]_{\mathbf{q}_{\mathrm{m}_{1}}-1}\right)\left[\mathrm{m}_{1}\right]_{\mathbf{q}_{\mathrm{m}_{1}}}^{2} \mathrm{q}_{\mathrm{m}_{1}}^{3}}{\left(1+\left[\mathrm{m}_{1}\right]_{\mathbf{q}_{\mathrm{m}_{1}}}\right)\left[\mathrm{m}_{1}+2\right]_{\mathbf{q}_{\mathrm{m}_{1}}}\left[\mathrm{~m}_{1}+3\right]_{\mathbf{q}_{\mathrm{m}_{1}}}}-\frac{2 \mathbf{q}_{\mathrm{m}_{1}}\left[\mathrm{~m}_{1}\right]_{\mathbf{q}_{\mathrm{m}_{1}}}}{\left[\mathrm{~m}_{1}+2\right]_{\mathbf{q}_{\mathrm{m}_{1}}}}\right) z_{1}^{2} \\
& +\left(\frac{2\left[m_{1}\right]_{\mathbf{q}_{m_{1}}}^{2} q_{m_{1}}^{3}}{\left(1+\left[m_{1}\right]_{\mathbf{q}_{m_{1}}}\right)\left[m_{1}+2\right]_{\mathbf{q}_{m_{1}}}\left[m_{1}+3\right]_{\mathbf{q}_{m_{1}}}}+\frac{\mathbf{q}_{m_{1}}\left(2 q_{m_{1}}+1\right)\left[m_{1}\right]_{\mathbf{q}_{m_{1}}}}{\left[m_{1}+2\right]_{\mathbf{q}_{m_{1}}}\left[m_{1}+3\right]_{\mathbf{q}_{m_{1}}}}-\frac{2}{\left[m_{1}+2\right]_{\mathbf{q}_{\mathrm{m}_{1}}}}\right) z_{1} \\
& +\frac{\mathbf{q}_{m_{1}}+1}{\left[m_{1}+2\right]_{q_{m_{1}}}\left[m_{1}+3\right]_{q_{m_{1}}}},
\end{aligned}
$$

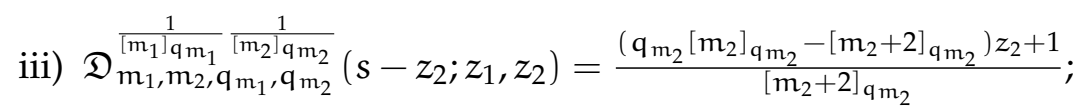

iv)

$$
\begin{aligned}
& \mathfrak{D}_{\mathrm{m}_{1}, \mathrm{~m}_{2}, \mathbf{q}_{\mathrm{m}_{1}}, \mathbf{q}_{\mathrm{m}_{2}}}^{\frac{1}{\left[\mathrm{~m}_{1}\right] \mathrm{q}_{\mathrm{m}_{2}}}}\left(\left(\mathrm{~s}-z_{2}\right)^{2} ; z_{1}, z_{2}\right) \\
& =\left(1+\frac{\mathbf{q}_{\mathrm{m}_{2}}\left(\left[\mathrm{~m}_{2}\right]_{\mathbf{q}_{\mathrm{m}_{2}}-1}\right)\left[\mathrm{m}_{2}\right]_{\mathbf{q}_{\mathrm{m}_{2}}}^{2} \mathrm{q}_{\mathrm{m}_{2}}^{3}}{\left(1+\left[\mathrm{m}_{2}\right]_{\mathbf{q}_{\mathrm{m}_{2}}}\right)\left[\mathrm{m}_{2}+2\right]_{\mathbf{q}_{\mathrm{m}_{2}}}\left[\mathrm{~m}_{2}+3\right]_{\mathbf{q}_{\mathrm{m}_{2}}}}-\frac{2 \mathbf{q}_{\mathrm{m}_{2}}\left[\mathrm{~m}_{2}\right]_{\mathbf{q}_{\mathrm{m}_{2}}}}{\left[\mathrm{~m}_{2}+2\right]_{\mathbf{q}_{\mathrm{m}_{2}}}}\right) z_{2}^{2} \\
& +\left(\frac{2\left[m_{2}\right]_{\mathbf{q}_{m_{2}}}^{2} q_{m_{2}}^{3}}{\left(1+\left[m_{2}\right]_{\mathfrak{q}_{m_{2}}}\right)\left[m_{2}+2\right]_{\mathfrak{q}_{m_{2}}}\left[m_{2}+3\right]_{\mathbf{q}_{m_{2}}}}+\frac{\mathbf{q}_{m_{2}}\left(2 q_{m_{2}}+1\right)\left[m_{2}\right]_{\mathbf{q}_{m_{2}}}}{\left[m_{1}+2\right]_{\mathfrak{q}_{m_{2}}}\left[m_{2}+3\right]_{\mathfrak{q}_{m_{2}}}}-\frac{2}{\left[m_{2}+2\right]_{\mathbf{q}_{m_{2}}}}\right) z_{2} \\
& +\frac{\mathrm{q}_{\mathrm{m}_{2}}+1}{\left[\mathrm{~m}_{2}+2\right]_{\mathrm{q}_{\mathrm{m}_{2}}}\left[\mathrm{~m}_{2}+3\right]_{\mathrm{q}_{\mathrm{m}_{2}}}} \text {. }
\end{aligned}
$$

Lemma 2.3. For $0<\mathrm{q}_{\mathrm{m}_{\mathrm{i}}}<1, i=1,2, z_{1}, z_{2} \in[0,1]$ and $\mathrm{m}_{1}, \mathrm{~m}_{2}>3$, we have

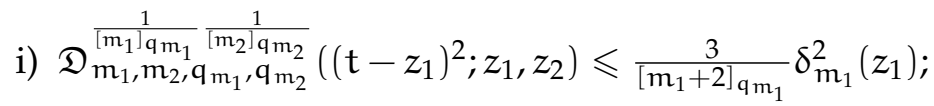

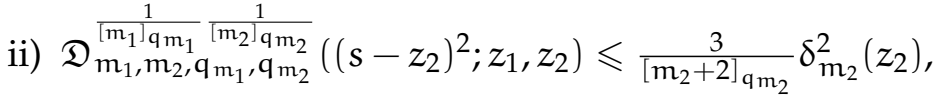

where $\delta_{\mathrm{m}_{1}}^{2}\left(z_{1}\right)=\psi^{2}\left(z_{1}\right)+\frac{3}{\left[\mathrm{~m}_{1}+2\right]_{\mathrm{q}_{1}}}, \psi^{2}\left(z_{1}\right)=z_{1}\left(1-z_{1}\right)$, and $\delta_{\mathrm{m}_{2}}^{2}\left(z_{2}\right)=\psi^{2}\left(z_{2}\right)+\frac{3}{\left[\mathrm{~m}_{2}+2\right]_{\mathrm{q}_{2}}}, \psi^{2}\left(z_{2}\right)=$ $z_{2}\left(1-z_{2}\right)$.

Corollary 2.4. Taking into account Lemma 2.2, we get the following limits as follows,

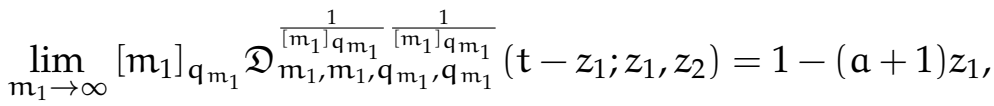

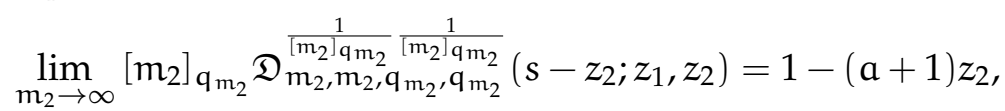

$$
\begin{aligned}
& \lim _{\mathfrak{m}_{1} \rightarrow \infty}\left[\mathrm{m}_{1}\right]_{\mathbf{q}_{\mathrm{m}_{1}}} \mathfrak{D}_{\mathrm{m}_{1}, \mathrm{~m}_{1}, \mathbf{q}_{\mathrm{m}_{1}}, \mathbf{q}_{\mathrm{m}_{1}}}^{\frac{1}{\left[\mathrm{~m}_{1}\right] \mathrm{q}_{1}}}\left(\left(\mathbf{t}-z_{1}\right)^{2} ; z_{1}, z_{2}\right)=3 z_{1}\left(1-z_{1}\right),
\end{aligned}
$$

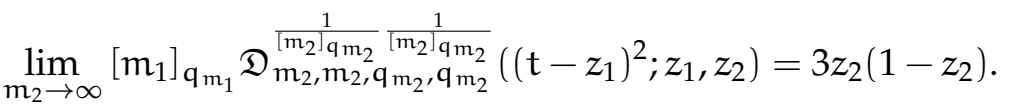




\section{Degree of approximation}

In what follow, we prove a convergence theorem for these operators, as well as Voronovskaja type theorem.

Theorem 3.1. Let $\left(q_{m_{i}}\right)$ be the sequences of real numbers such that $0<q_{m_{i}}<1$, and $\lim _{m_{i} \rightarrow \infty} q_{m_{i}}=1, i=1,2$. Then, the sequences $\left.\left\{\mathfrak{D}_{\mathrm{m}_{1}, \mathrm{~m}_{2}, \mathbf{q}_{\mathrm{m}_{1}}, \mathbf{q}_{\mathrm{m}_{2}}}^{\frac{1}{\left[\mathrm{~m}_{1}\right]}} \frac{1}{[\mathrm{~m} ;}, z_{1}, z_{2}\right)\right\}$ converge to $\mathrm{h}\left(z_{1}, z_{2}\right)$ uniformly on $\mathrm{I}^{2}$ for each $\mathrm{h} \in \mathrm{C}\left(\mathrm{I}^{2}\right)$.

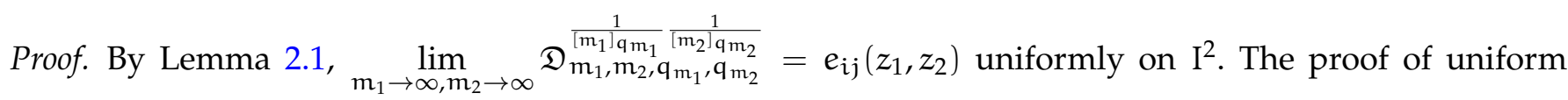
convergence is then completed by applying a Volkov Theorem [18].

Theorem 3.2. For $\mathrm{h} \in \mathrm{C}\left(\mathrm{I}^{2}\right)$, we have

$$
\begin{aligned}
& \lim _{m_{1} \rightarrow \infty}\left[m_{1}\right]_{\mathbf{q}_{m_{1}}}\left(\mathfrak{D}_{m_{1}, m_{1}, \mathbf{q}_{m_{1}}, \mathbf{q}_{m_{1}}}^{\frac{1}{\left[m_{1} q_{m_{1}}\right.}} \frac{1}{\left[m_{1}\right]}\left(z_{1}, z_{2}\right)-h\left(z_{1}, z_{2}\right)\right) \\
& =\left(1-(\mathrm{a}+1) z_{1}\right) \mathrm{h}_{z_{1}}^{\prime}\left(z_{1}, z_{2}\right)+\left(1-(\mathrm{a}+1) z_{2}\right) \mathrm{h}_{z_{2}}^{\prime}\left(z_{1}, z_{2}\right)+\frac{3}{2}\left\{z_{1}\left(1-z_{1}\right) h_{z_{1} z_{1}}\left(z_{1}, z_{2}\right)+z_{2}\left(1-z_{2}\right) h_{z_{2} z_{2}}\left(z_{1}, z_{2}\right)\right\},
\end{aligned}
$$

uniformly on $\mathrm{I}^{2}$.

Proof. For $z_{1}, z_{2} \in \mathrm{I}^{2}$, by the Taylor's expansion formula, we have

$$
\begin{aligned}
h(t, s)= & h\left(z_{1}, z_{2}\right)+h_{z_{1}}^{\prime}\left(z_{1}, z_{2}\right)\left(t-z_{1}\right)+h_{z_{2}}^{\prime}\left(z_{1}, z_{2}\right)\left(s-z_{2}\right) \\
& +\frac{1}{2}\left\{h_{z_{1} z_{1}}^{\prime \prime}\left(z_{1}, z_{2}\right)\left(t-z_{1}\right)^{2}+2 h_{z_{1} z_{2}}\left(z_{1}, z_{2}\right)\left(t-z_{1}\right)\left(s-z_{2}\right)+h_{z_{2} z_{2}}^{\prime \prime}\left(z_{1}, z_{2}\right)\left(s-z_{2}\right)^{2}\right\} \\
& +\psi\left(t, s ; z_{1}, z_{2}\right)\left(\left(t-z_{1}\right)^{2}+\left(t-z_{2}\right)^{2}\right),
\end{aligned}
$$

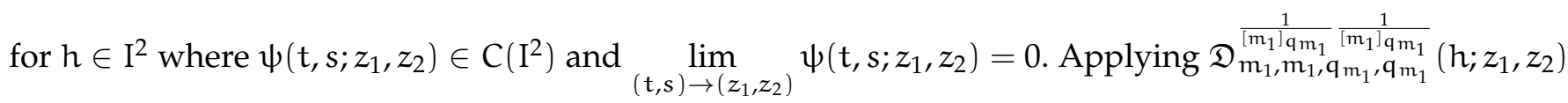
on above Taylor's expansion formula, we can write

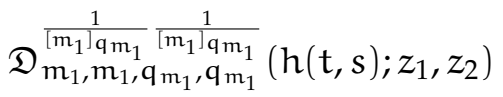

$$
\begin{aligned}
& =h\left(z_{1}, z_{2}\right)+h_{z_{1}}^{\prime}\left(z_{1}, z\right) \mathfrak{D}_{\mathrm{m}_{1}, \mathrm{~m}_{2}, \mathbf{q}_{\mathrm{m}_{1}}, \mathrm{q}_{\mathrm{m}_{2}}}^{\frac{1}{\left[\mathrm{~m}_{1} \mathrm{~m}_{\mathrm{m}_{1}}\right.}}\left(\left(\mathrm{t}-z_{1}\right) ; z_{1}, z_{2}\right)
\end{aligned}
$$

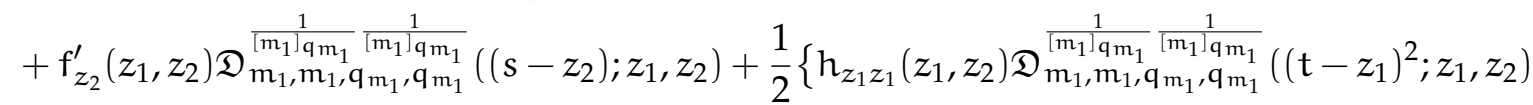

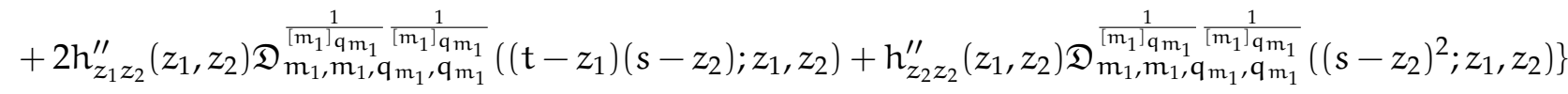

$$
\begin{aligned}
& +\psi\left(t, s ; z_{1}, z_{2}\right) \mathfrak{D}_{m_{1}, m_{1}, q_{m_{1}}, q_{m_{1}}}^{\frac{1}{\left[m_{1} q_{m_{1}}\right.} \frac{1}{m_{1} q_{m_{1}}}}\left(\left(\left(t-z_{1}\right)^{2}+\left(t-z_{2}\right)^{2}\right) ; z_{1}, z_{2}\right) \\
& =h\left(z_{1}, z_{2}\right)+h_{z_{1}}^{\prime}\left(z_{1}, z_{2}\right) \mathfrak{D}_{m_{1}, q_{m_{1}}}^{\frac{1}{\left[m_{1}\right] \mathrm{q}_{1}}}\left(\left(t-z_{1}\right) ; z_{1}, z_{2}\right) \\
& +\mathrm{h}_{z_{2}}^{\prime}\left(z_{1}, z_{2}\right) \mathfrak{D}_{\mathrm{m}_{1}, \mathbf{q}_{\mathrm{m}_{1}}}^{\frac{1}{\left[\mathrm{~m}_{1}\right] \mathrm{q}_{1}}}\left(\left(\mathrm{~s}-z_{2}\right) ; z_{1}, z_{2}\right)+\frac{1}{2}\left\{h_{z_{1} z_{1}}^{\prime \prime}\left(z_{1}, z_{2}\right) \mathfrak{D}_{\mathrm{m}_{1}, \mathbf{q}_{\mathrm{m}_{1}}}^{\frac{1}{\left[\mathrm{~m}_{1}\right] \mathrm{m}_{1}}}\left(\left(\mathrm{t}-z_{1}\right)^{2} ; z_{1}, z_{2}\right)\right. \\
& +2 \mathrm{~h}_{z_{1} z_{2}}^{\prime \prime}\left(z_{1}, z_{2}\right) \mathfrak{D}_{m_{1}, q_{m_{1}}}^{\frac{1}{\left[m_{1} q_{m_{1}}\right.}}\left(\left(t-z_{1}\right) ; z_{1}, z_{2}\right) \mathfrak{D}_{m_{1}, q_{m_{1}}}^{\frac{1}{\left[m_{1}\right] \mathrm{q}_{1}}}\left(\left(s-z_{2}\right) ; z_{1}, z_{2}\right)
\end{aligned}
$$

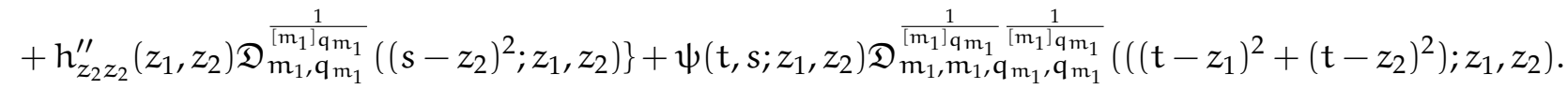


Using the Cauchy-Schwarz inequality, we have

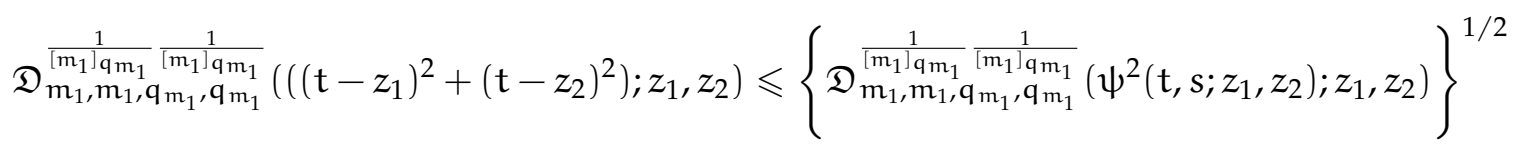

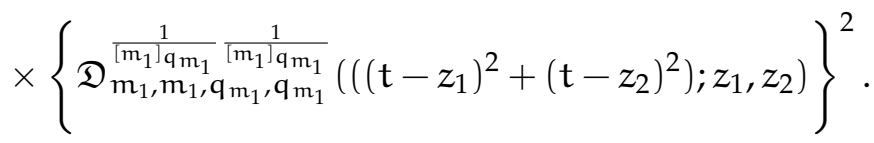

Hence, by using Theorem 2.4, we get

$$
\begin{aligned}
\lim _{\mathrm{m}_{1} \rightarrow \infty}\left[\mathrm{m}_{1}\right]_{\mathbf{q}_{\mathrm{m}_{1}}} & \left(\mathfrak{D}_{\mathrm{m}_{1}, \mathrm{~m}_{1}, \mathbf{q}_{\mathrm{m}_{1}}, \mathbf{q}_{\mathrm{m}_{1}}}^{\frac{1}{\left[\mathrm{~m}_{1}\right]} \frac{1}{\left[m_{1}\right] \mathrm{q}_{1}}}\left(h ; z_{1}, z_{2}\right)-h\left(z_{1}, z_{2}\right)\right) \\
= & \left(1-(\mathrm{a}+1) z_{1}\right) \mathrm{f}_{z_{1}}^{\prime}\left(z_{1}, z_{2}\right)+\left(1-(\mathrm{a}+1) z_{2}\right) \mathrm{h}_{z_{2}}^{\prime}\left(z_{1}, z_{2}\right) \\
& +\frac{3}{2}\left\{z_{1}\left(1-z_{1}\right) h_{z_{1} z_{1}}^{\prime \prime}\left(z_{1}, z_{2}\right)+z_{2}\left(1-z_{2}\right) h_{z_{2} z_{2}}^{\prime \prime}\left(z_{1}, z_{2}\right)\right\},
\end{aligned}
$$

which leads us to the required result.

For $h \in \mathrm{C}\left(\mathrm{I}^{2}\right)$ and $\delta>0$, the complete modulus of continuity for the function $h\left(z_{1}, z_{2}\right)$ is defined by

$$
\omega\left(h ; \delta_{1}, \delta_{2}\right)=\sup \left\{\left|h(u, v)-h\left(z_{1}, z_{2}\right)\right|:(u, v),\left(z_{1}, z_{2}\right) \in \mathrm{I}^{2},\left|u-z_{1}\right| \leqslant \delta_{1},\left|v-z_{2}\right| \leqslant \delta_{2}\right\},
$$

and its partial modulus of continuity with respect to $z_{1}$ and $z_{2}$ are respectively given by

$$
\begin{aligned}
& \omega^{(1)}(h ; \delta)=\sup _{0 \leqslant z_{2} \leqslant b} \sup _{\left|x_{1}-x_{2}\right| \leqslant \delta}\left\{\left|h\left(x_{1}, z_{2}\right)-h\left(x_{2}, z_{2}\right)\right|\right\}, \\
& \omega^{(2)}(h ; \delta)=\sup _{0 \leqslant z_{1} \leqslant a} \sup _{\left|y_{1}-y_{2}\right| \leqslant \delta}\left\{\left|h\left(z_{1}, y_{1}\right)-f\left(z_{1}, y_{2}\right)\right|\right\} .
\end{aligned}
$$

Now, we obtain the rate of convergence of the approximation by the bivariate operators (2.3) defined by means of modulus of continuity of the functions.

Theorem 3.3. Let $\mathrm{h} \in \mathrm{C}\left(\mathrm{I}^{2}\right)$ and $\left(\mathrm{q}_{\mathrm{m}_{\mathrm{i}}}\right)$ be a sequence of real numbers such that $0<\mathrm{q}_{\mathrm{m}_{\mathrm{i}}}<1$, and $\lim _{\mathrm{m}_{\mathrm{i}} \rightarrow \infty} \mathrm{q}_{\mathrm{m}_{\mathrm{i}}}=$ $1, i=1,2$. Then, for all $\left(z_{1}, z_{2}\right) \in I^{2}$, we have

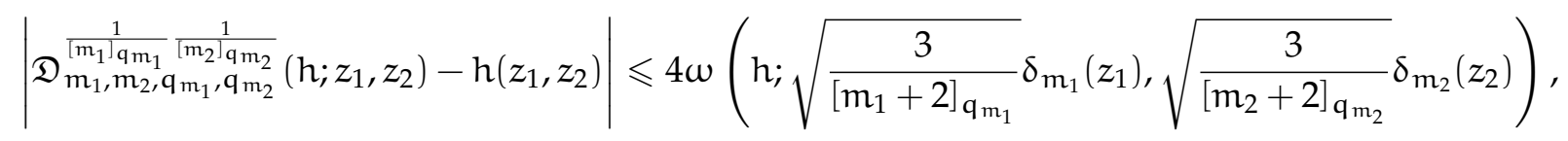

where $\delta_{\mathrm{m}_{1}}\left(z_{1}\right)$ and $\delta_{\mathrm{m}_{2}}\left(z_{2}\right)$ are defined by Lemma 2.3.

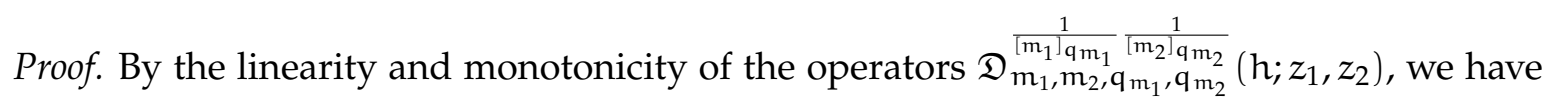

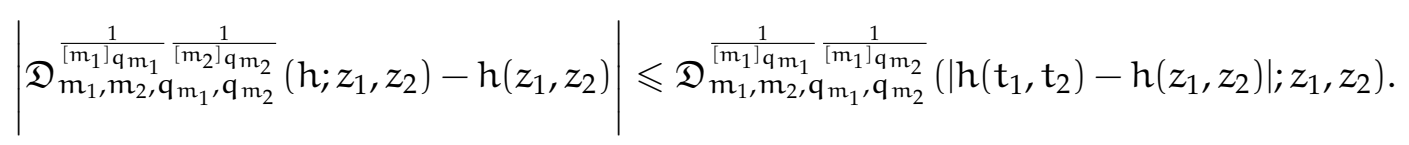

Taking into account the property of monotonicity of $\omega\left(h ; \delta_{m_{1}}, \delta_{m_{2}}\right)$, we get

$$
\begin{aligned}
& \left|\mathfrak{D} \frac{1}{\frac{1}{m_{m_{1}}, \mathrm{q}_{2}, \mathrm{q}_{\mathrm{m}_{1}}} \frac{1}{\left.\mathrm{~m}_{2}\right] \mathrm{q}_{\mathrm{m}_{2}}}}\left(\mathrm{~h} ; z_{1}, z_{2}\right)-\mathrm{h}\left(z_{1}, z_{2}\right)\right| \\
& \leqslant \omega\left(h ; \sqrt{\delta_{m_{1}}\left(z_{1}\right)}, \sqrt{\delta_{m_{2}}\left(z_{1}\right)}\right)\left(\mathfrak{D}_{m_{1}, m_{2}, q_{m_{1}}, q_{m_{2}}}^{\frac{1}{\left[m_{1}\right] q_{m_{1}}} \frac{1}{\left.m_{2}\right]}}\left(1 ; z_{1}, z_{2}\right)+\frac{1}{\sqrt{\delta_{m_{1}}\left(z_{1}\right)}} \mathfrak{D}_{m_{1}, m_{2}, q_{m_{1}}, q_{m_{2}}}^{\frac{1}{\left[m_{1}\right] q_{m_{1}}} \frac{1}{\left.m_{2}\right]}}\left(\left|t_{1}-z_{1}\right| ; z_{1}, z_{2}\right)\right)
\end{aligned}
$$




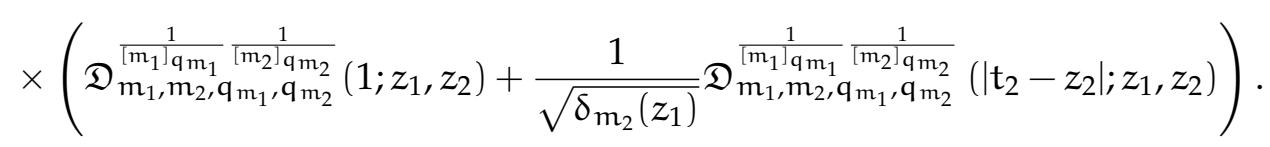

Then, by using the Cauchy-Schwarz inequality, we have

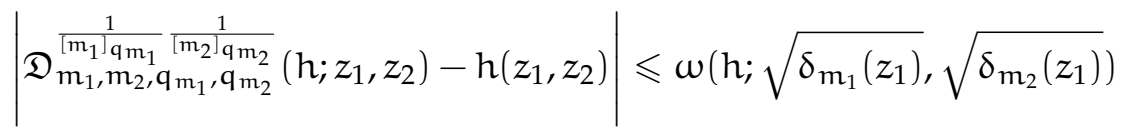

$$
\begin{aligned}
& \times\left(1+\frac{1}{\sqrt{\delta_{m_{1}}}}\left\{\mathfrak{D}_{m_{1}, q_{\mathrm{m}_{1}}}^{\frac{1}{\left[m_{1}\right] \mathrm{q}_{\mathrm{m}_{1}}}}\left(\left(\mathrm{t}_{1}-z_{1}\right)^{2} ; z_{1}, z_{2}\right)\right\}^{1 / 2}\right) \\
& \times\left(1+\frac{1}{\sqrt{\delta_{m_{2}}}}\left\{\mathfrak{D}_{m_{2}, q_{m_{2}}}^{\frac{1}{m_{2} \mathfrak{q}_{m_{2}}}}\left(\left(t_{2}-z_{2}\right)^{2} ; z_{1}, z_{2}\right)\right\}^{1 / 2}\right) \text {. }
\end{aligned}
$$

Finally, choosing $\delta_{\mathrm{m}_{1}}=\delta_{\mathrm{m}_{1}}\left(z_{1}\right)$ and $\delta_{\mathrm{m}_{2}}=\delta_{\mathrm{m}_{2}}\left(z_{2}\right)$, we reach the desired result.

We continue by recalling the definition of the Lipschitz class for bivariate function of $\mathrm{f}$. For $0<\gamma \leqslant 1$, the Lipschitz class $\operatorname{Lip}_{\mathrm{L}}(\gamma)$ for bivariate case is as follows:

$$
\operatorname{Lip}_{\mathrm{L}}(\gamma):=\left\{h:\left|h\left(\mathrm{t}_{1}, \mathrm{t}_{2}\right)-\mathrm{h}\left(z_{1}, z_{2}\right)\right| \leqslant \mathrm{L} \frac{\|\mathrm{r}-\mathrm{s}\|^{\gamma}}{\left(\|\mathrm{r}\|+z_{1}+z_{2}\right)^{\gamma / 2}}\right\},
$$

where $r=\left(t_{1}, t_{2}\right), s=\left(z_{1}, z_{2}\right)$ in $I^{2}$ and $\|r-s\|=\left\{\left(t_{1}-z_{1}\right)^{2}+\left(t_{2}-z_{2}\right)^{2}\right\}^{1 / 2}$ is the Euclidean norm.

Theorem 3.4. Suppose that $\mathrm{h} \in \operatorname{Lip}_{\mathrm{L}}(\gamma)$. Then, for every $\left(z_{1}, z_{2}\right) \in \mathrm{I}^{2}$, we have

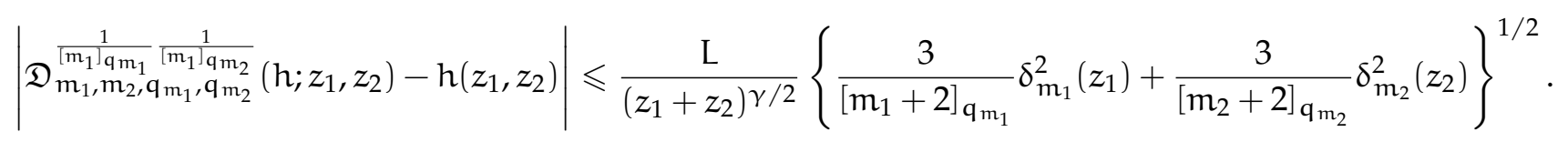

Proof. Take $\gamma=1$. Then, for $\mathrm{h} \in \operatorname{Lip}_{\mathrm{L}}(\gamma)$ and for each $z_{1}, z_{2} \in \mathrm{I}^{2}$, using the monotonicity and linearity of operators, we have

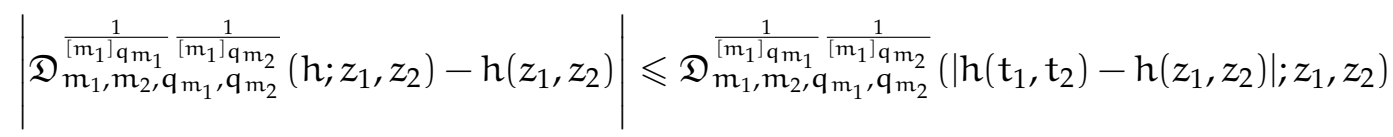

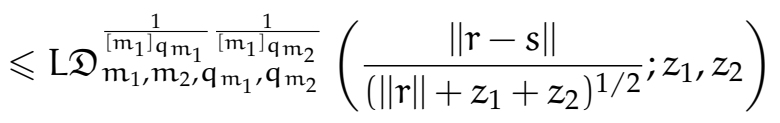

$$
\begin{aligned}
& \leqslant \frac{\mathrm{L}}{\left(z_{1}+z_{2}\right)^{1 / 2}} \mathfrak{D}_{\mathrm{m}_{1}, \mathrm{~m}_{2}, \mathbf{q}_{\mathrm{m}_{1}}, \mathbf{q}_{\mathrm{m}_{2}}}^{\frac{1}{\left[\mathrm{~m}_{1}\right]}} \frac{1}{\left[\mathrm{~m}_{1} \mathrm{q}_{\mathrm{m}}\right.}\left(\|\mathrm{r}-\mathrm{s}\| ; z_{1}, z_{2}\right),
\end{aligned}
$$

where $r=\left(t_{1}, t_{2}\right)$ and $s=\left(z_{1}, z_{2}\right)$. Using the Cauchy-Schwarz inequality and Lemma 2.1, we obtain

$$
\begin{aligned}
& \left|\mathfrak{D} \frac{1}{\frac{1}{\left[m_{1}\right] \mathfrak{q}_{1},} \frac{1}{\left[m_{1}\right] \mathfrak{q}_{2}}}\left(h ; z_{1}, z_{2}\right)-h\left(z_{1}, z_{2}\right)\right| \\
& \leqslant \frac{\mathrm{L}}{\left(z_{1}+z_{2}\right)^{1 / 2}}\left\{\mathfrak{D}_{\mathrm{m}_{1}, \mathrm{~m}_{2}, \mathbf{q}_{\mathrm{m}_{1}}, \mathbf{q}_{\mathrm{m}_{2}}}^{\frac{1}{\left[\mathrm{~m}_{1} \mathrm{q}_{\mathrm{m}_{2}}\right.} \frac{1}{\left[\mathrm{n}_{1} \mathrm{q}_{\mathrm{m}_{2}}\right.}}\left(\|\mathrm{r}-\mathrm{s}\|^{2}, z_{1}, z_{2}\right)\right\}^{1 / 2} \\
& \leqslant \frac{\mathrm{L}}{\left(z_{1}+z_{2}\right)^{1 / 2}}\left\{\mathfrak{D}_{\mathrm{m}_{1}, \mathbf{q}_{\mathrm{m}_{1}}}^{\frac{1}{\left[\mathrm{~m}_{1} \mathrm{q}_{\mathrm{m}_{1}}\right.}}\left(\left(\mathrm{t}_{1}-z_{1}\right)^{2}, z_{1}, z_{2}\right)+\mathfrak{D}_{\mathrm{m}_{2}, \mathbf{q}_{\mathrm{m}_{2}}}^{\frac{1}{\left[\mathrm{~m}_{2}\right] \mathrm{q}_{\mathrm{m}_{2}}}}\left(\left(\mathrm{t}_{2}-z_{2}\right)^{2}, z_{1}, z_{2}\right)\right\}^{1 / 2}
\end{aligned}
$$




$$
\leqslant \frac{L}{\left(z_{1}+z_{2}\right)^{1 / 2}}\left\{\frac{3}{\left[m_{1}+2\right]_{\mathfrak{q}_{1}}} \delta_{m_{1}}^{2}\left(z_{1}\right)+\frac{3}{\left[m_{2}+2\right]_{\mathrm{q}_{2}}} \delta_{\mathrm{m}_{2}}^{2}\left(z_{2}\right)\right\}^{1 / 2} .
$$

Secondly, let $0<\gamma<1$. Then, for $h \in \operatorname{Lip}_{\mathrm{L}}(\gamma)$ and for each $z_{1}, z_{2} \in \mathrm{I}^{2}$, we get

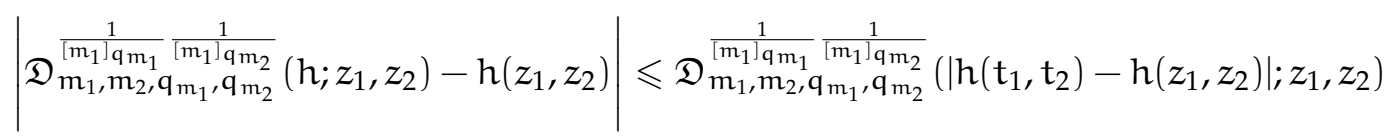

$$
\begin{aligned}
& \leqslant \operatorname{LD}_{\mathfrak{m}_{1}, m_{2}, q_{m_{1}}, q_{m_{2}}}^{\frac{1}{\left[m_{1}\right] q_{m_{1}}}} \frac{1}{\left[m_{1} q_{m_{2}}\right.}\left(\frac{\|r-s\|^{\gamma}}{\left(\|r\|+z_{1}+z_{2}\right)^{\gamma / 2}} ; z_{1}, z_{2}\right) \\
& \leqslant \frac{\mathrm{L}}{\left(z_{1}+z_{2}\right)^{\gamma / 2}} \mathfrak{D}_{\mathrm{m}_{1}, \mathrm{~m}_{2}, \mathbf{q}_{\mathrm{m}_{1}}, \mathbf{q}_{\mathrm{m}_{2}}}^{\frac{1}{\left[\mathrm{~m}_{1} \mathbf{q}_{\mathrm{m}_{2}}\right.}} \frac{1}{\left[\mathrm{~m}_{1} \mathrm{q}_{\mathrm{m}_{2}}\right.}\left(\|\mathrm{r}-\mathrm{s}\|^{\gamma} ; z_{1}, z_{2}\right) \text {. }
\end{aligned}
$$

Now, applying the Hölder's inequality with $\mathfrak{u}_{1}=\frac{2}{\gamma}$ and $u_{2}=\frac{2}{2-\gamma}$, and Lemma 2.1, we get

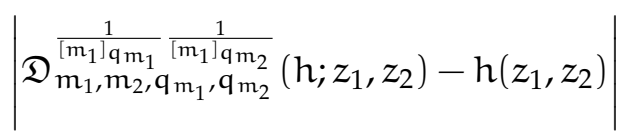

$$
\begin{aligned}
& \leqslant \frac{L}{\left(z_{1}+z_{2}\right)^{\gamma / 2}}\left\{\mathfrak{D}_{m_{1}, m_{2}, q_{m_{1}}, q_{m_{2}}}^{\frac{1}{\left[m_{1}\right] q_{m_{2}}} \frac{1}{\left[m_{1}\right] q_{m_{2}}}}\left(\|r-s\|^{2}, z_{1}, z_{2}\right)\right\}^{\gamma / 2} \\
& \leqslant \frac{\mathrm{L}}{\left(z_{1}+z_{2}\right)^{\gamma / 2}}\left\{\mathfrak{D}_{\mathrm{m}_{1}, \mathbf{q}_{\mathrm{m}_{1}}}^{\frac{1}{\mathrm{~m}_{1} \mathbf{q}_{\mathrm{m}_{1}}}}\left(\left(\mathrm{t}_{1}-z_{1}\right)^{2}, z_{1}, z_{2}\right)+\mathfrak{D}_{\mathrm{m}_{2}, \mathbf{q}_{\mathrm{m}_{2}}}^{\frac{1}{\left[\mathrm{~m}_{2}\right] \mathrm{m}_{2}}}\left(\left(\mathrm{t}_{2}-z_{2}\right)^{2}, z_{1}, z_{2}\right)\right\}^{\gamma / 2} \\
& \leqslant \frac{L}{\left(z_{1}+z_{2}\right)^{1 / 2}}\left\{\frac{3}{\left[m_{1}+2\right]_{q_{m_{1}}}} \delta_{m_{1}}^{2}\left(z_{1}\right)+\frac{3}{\left[m_{2}+2\right]_{q_{m_{2}}}} \delta_{m_{2}}^{2}\left(z_{2}\right)\right\}^{\gamma / 2},
\end{aligned}
$$

which leads us to the required result.

Theorem 3.5. Suppose that $\mathrm{h} \in \mathrm{C}^{1}\left(\mathrm{I}^{2}\right)$. Then, for every $\left(z_{1}, z_{2}\right) \in \mathrm{I}^{2}$, we have

$$
\left.\mid \mathfrak{D}_{m_{1}, m_{2}, \mathbf{q}_{m_{1}}, \mathbf{q}_{m_{2}}}^{\frac{1}{\left[m_{1}\right] m_{1}}} \frac{1}{\left[m_{m_{2}}\right.} z_{1}, z_{2}\right)-h\left(z_{1}, z_{2}\right) \mid \leqslant\left\|h_{z_{1}}^{\prime}\right\|_{C\left(I_{a b}\right)} \sqrt{\delta_{m_{1}}\left(z_{1}\right)}+\left\|h_{z_{2}}^{\prime}\right\|_{C\left(I_{a b}\right)} \sqrt{\delta_{m_{2}}\left(z_{2}\right)},
$$

where $\delta_{\mathrm{m}_{1}}\left(z_{1}\right)$ and $\delta_{\mathrm{m}_{2}}\left(z_{2}\right)$ are defined by Lemma 2.3.

Proof. For $\left(z_{1}, z_{2}\right) \in \mathrm{I}^{2}$ be a fixed point, we obtain,

$$
h\left(t_{1}, t_{2}\right)-h\left(z_{1}, z_{2}\right)=\int_{z_{1}}^{t_{1}} h_{r_{1}}^{\prime}\left(r_{1}, t_{2}\right) d r_{1}+\int_{z_{2}}^{t_{2}} h_{r_{2}}^{\prime}\left(z_{1}, r_{2}\right) d r_{2}, \text { for }\left(t_{1}, t_{2}\right) \in I^{2} .
$$

Applying the operator defined in (2.3) on both sides, we obtain

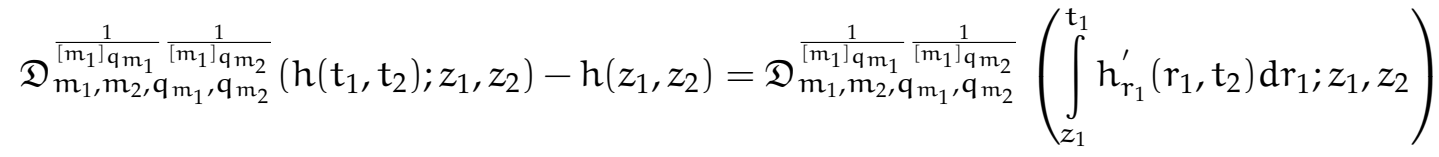

$$
\begin{aligned}
& +\mathfrak{D}_{m_{1}, m_{2}, q_{m_{1}}, q_{m_{2}}}^{\frac{1}{\left[m_{1}\right] m_{m_{2}}}} \frac{1}{m_{m_{1}} \mathfrak{q}_{m_{2}}}\left(\int_{z_{2}}^{t_{2}} h_{r_{2}}^{\prime}\left(z_{1}, r_{2}\right) d r_{2} ; z_{1}, z_{2}\right) \text {. }
\end{aligned}
$$


Now, by using sup-norm on $\mathrm{I}^{2}$, we get

$$
\left|\int_{z_{1}}^{t_{1}} h_{r_{1}}^{\prime}\left(r_{1}, t_{2}\right) d r_{1}\right| \leqslant \int_{z_{1}}^{t_{1}}\left|h_{r_{1}}^{\prime}\left(r_{1}, t_{2}\right)\right|\left|d r_{1}\right| \leqslant\left\|h_{z_{1}}^{\prime}\right\|_{C_{\left(I^{2}\right)}}\left|t_{1}-z_{1}\right|
$$

and

$$
\left|\int_{z_{2}}^{t_{1}} h_{r_{2}}^{\prime}\left(z_{1}, r_{2}\right) d r_{2}\right| \leqslant \int_{z_{2}}^{t_{2}}\left|h_{r_{2}}^{\prime}\left(z_{1}, r_{2}\right)\right|\left|d r_{2}\right| \leqslant\left\|h_{z_{2}}^{\prime}\right\| c_{\left(1^{2}\right)}\left|t_{2}-z_{2}\right| .
$$

By using these inequalities, we have

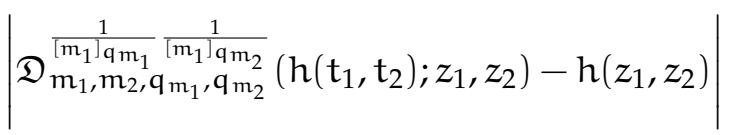

$$
\begin{aligned}
& \leqslant \mathfrak{D}_{m_{1}, m_{2}, \mathbf{q}_{m_{1}}, \mathbf{q}_{m_{2}}}^{\frac{1}{\left[m_{1}\right] m_{1}}} \frac{1}{\left[m_{1}\right] m_{2}}\left(\left|\int_{z_{1}}^{t_{1}} h_{r_{1}}^{\prime}\left(r_{1}, t_{2}\right) d t\right| ;, z_{1}, z_{2}\right)+\mathfrak{D}_{m_{1}, m_{2}, \mathbf{q}_{m_{1}}, \mathbf{q}_{m_{2}}}^{\frac{1}{\left[m_{1}\right] m_{1}}} \frac{1}{\left[m_{1}\right] m_{2}}\left(\left|\int_{z_{2}}^{t_{2}} h_{r_{2}}^{\prime}\left(z_{1}, r_{2}\right) d r_{2}\right| ; z_{1}, z_{2}\right) \\
& \leqslant\left\|h_{z_{1}}^{\prime}\right\|_{I^{2}} \mathfrak{D}_{m_{1}, \mathbf{q}_{\mathrm{m}_{1}}}^{\frac{1}{m_{1} \mathfrak{m}_{1}}}\left(\left|t_{1}-z_{1}\right| ; z_{1}, z_{2}\right)+\left\|h_{z_{2}}^{\prime}\right\|_{\mathrm{I}^{2}} \mathfrak{D}_{m_{2}, \mathbf{q}_{\mathrm{m}_{2}}}^{\frac{1}{\left[\mathrm{~m}_{2} / \mathrm{q}_{2}\right.}}\left(\left|\mathrm{t}_{2}-z_{2}\right| ; z_{1}, z_{2}\right) .
\end{aligned}
$$

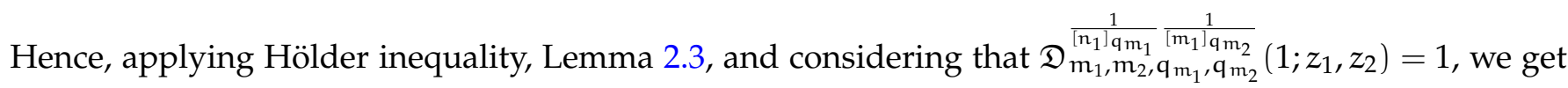

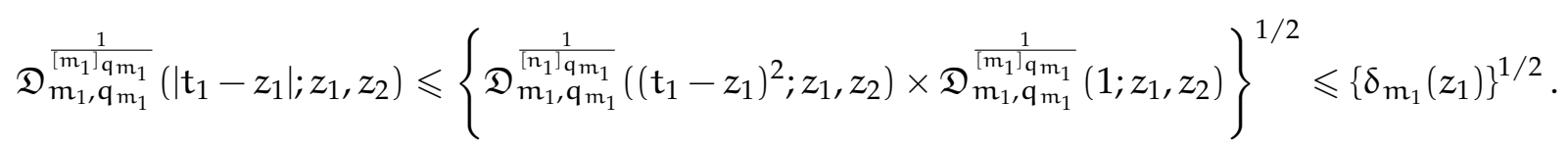

Analogously,

$$
\mathfrak{D}_{\mathrm{m}_{2}, \mathbf{q}_{\mathrm{m}_{2}}}^{\frac{1}{\left[\mathrm{~m}_{2}\right] \mathrm{q}_{2}}}\left(\left|\mathrm{t}_{2}-z_{2}\right| ; z_{1}, z_{2}\right) \leqslant\left\{\mathfrak{D}_{\mathrm{m}_{2}, \mathbf{q}_{\mathrm{m}_{2}}}^{\frac{1}{\left[\mathrm{~m}_{2} \mathrm{q}_{2}\right.}}\left(\left(\mathrm{t}_{2}-z_{2}\right)^{2} ; z_{1}, z_{2}\right) \times \mathfrak{D}_{\mathrm{m}_{2}, \mathbf{q}_{\mathrm{m}_{2}}}^{\frac{1}{\left.\mathrm{~m}_{2}\right] \mathrm{q}_{2}}}\left(1 ; z_{1}, z_{2}\right)\right\}^{1 / 2} \leqslant\left\{\delta_{\mathrm{m}_{2}}\left(z_{2}\right)\right\}^{1 / 2}
$$

Combining equations (3.1)-(3.3), we obtain

$$
\left|\mathfrak{D}_{m_{1}, m_{2}, q_{m_{1}}, q_{m_{2}}}^{\frac{1}{m_{1} \mid m_{m_{1}}} \frac{1}{\left.m_{1}\right] q_{m_{2}}}}\left(h ; z_{1}, z_{2}\right)-h\left(z_{1}, z_{2}\right)\right| \leqslant\left\|h_{z_{1}}^{\prime}\right\|_{C\left(I^{2}\right)} \sqrt{\delta_{m_{1}}\left(z_{1}\right)}+\left\|h_{z_{2}}^{\prime}\right\|_{C\left(I^{2}\right)} \sqrt{\delta_{m_{2}}\left(z_{2}\right)} .
$$

This completes the proof.

Let $C^{2}\left(I_{a b}\right)$ be the space of functions $h$ such that $\frac{\partial^{i} h}{\partial z_{1} i}, \frac{\partial^{i} h}{\partial z_{2}{ }^{i}} \in C\left(I_{a b}\right),(i=1,2)$. The norm on the space $\mathrm{C}^{2}\left(\mathrm{I}_{\mathrm{ab}}\right)$ is defined as

$$
\|h\|_{C^{2}\left(I_{a b}\right)}=\|h\|_{C\left(I_{a b}\right)}+\sum_{i=1}^{2}\left(\left\|\frac{\partial^{i} h}{\partial z_{1}^{i}}\right\|_{C\left(I_{a b}\right)}+\left\|\frac{\partial^{i} h}{\partial z_{2}^{i}}\right\|_{C\left(I_{a b}\right)}\right) .
$$

For $h \in C\left(I_{a b}\right)$ and $\delta>0$, the Peetre's K-functional and the second modulus of smoothness are defined respectively as

$$
\mathrm{K}(\mathrm{h} ; \delta)=\inf _{\mathrm{g} \in \mathrm{c}^{2}\left(\mathrm{I}_{\mathrm{ab}}\right)}\left\{\|\mathrm{h}-\mathrm{g}\|_{\mathrm{C}\left(\mathrm{I}_{\mathrm{ab}}\right)}+\delta\|\mathrm{g}\|_{\mathrm{C}\left(\mathrm{I}_{\mathrm{ab}}\right)}\right\}
$$

and

$$
\omega_{2}(h ; \delta)=\sup _{\sqrt{u^{2}+v^{2}} \leqslant \delta}\left\|h\left(z_{1}+2 u, z_{2}+2 v\right)-2 h\left(z_{1}+u, z_{2}+v\right)+h\left(z_{1}, z_{2}\right)\right\|_{c\left(I_{a b}\right)}
$$


where $\|\cdot\|_{\mathrm{C}\left(\mathrm{I}_{\mathrm{ab}}\right)}$ is the sup-norm. It is known that ([6], page 192) there exists a positive constant $L$ that is independent of $\delta$ and $h$, such that

$$
\mathrm{K}(\mathrm{h} ; \delta) \leqslant \mathrm{L}\left\{\omega_{2}(\mathrm{~h} ; \delta)+\min (1, \delta)\|\mathrm{h}\|_{\mathrm{C}\left(\mathrm{I}_{\mathrm{ab}}\right)}\right\} .
$$

Below, we prove the following order of approximation of q-Durrmeyer-Pólya operators to the function $h \in \mathrm{C}^{2}\left(\mathrm{I}^{2}\right)$ by K-functional.

Theorem 3.6. Let $\mathrm{h} \in \mathrm{C}\left(\mathrm{I}^{2}\right)$. Then we have estimates

$$
\begin{aligned}
& \mathfrak{D}_{\mathrm{m}_{1}, \mathrm{~m}_{2}, \mathbf{q}_{\mathrm{m}_{1}}, \mathbf{q}_{\mathrm{m}_{2}}}^{\frac{1}{\left[\mathrm{~m}_{2}\right] \mathrm{q}_{\mathrm{m}_{2}}} \frac{1}{\mathrm{~m}_{\mathrm{m}_{2}}}}\left(\mathrm{~h} ; z_{1}, z_{2}\right)-\mathrm{h}\left(z_{1}, z_{2}\right) \\
& \leqslant L\left\{w_{2}\left(h ; \sqrt{\lambda_{m_{1}, m_{2}, p_{1}, p_{2}}\left(q_{n_{1}}, q_{n_{2}}, z_{1}, z_{2}\right)}\right)\right. \\
& \left.+\min \left\{1, \eta_{m_{1}, m_{2}, p_{1}, p_{2}}\left(q_{m_{1}}, q_{m_{2}}, z_{1}, z_{2}\right)\right\}\|h\|_{C\left(I_{a b}\right)}\right\}+\omega\left(h ; \sqrt{\left(u_{m_{1}}-z_{1}\right)^{2}+\left(v_{m_{2}}-z_{2}\right)^{2}}\right),
\end{aligned}
$$

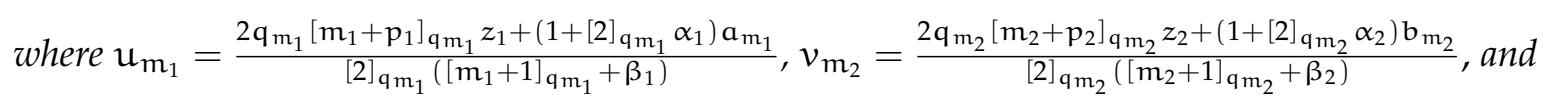

$$
\eta_{m_{1}, m_{2}}\left(h ; q_{m_{1}}, q_{m_{2}}, z_{1}, z_{2}\right)=\delta_{m_{1}}+\delta_{m_{2}}+\left(u_{m_{1}}-z_{1}\right)^{2}+\left(v_{m_{2}}-z_{2}\right)^{2}
$$

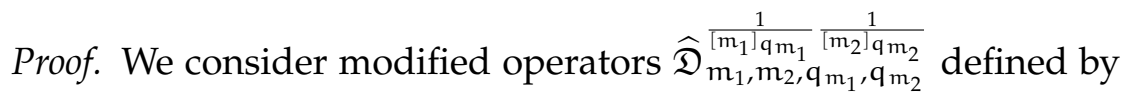

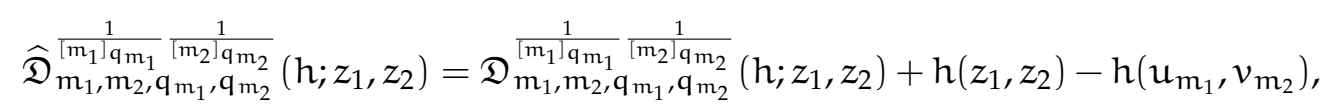

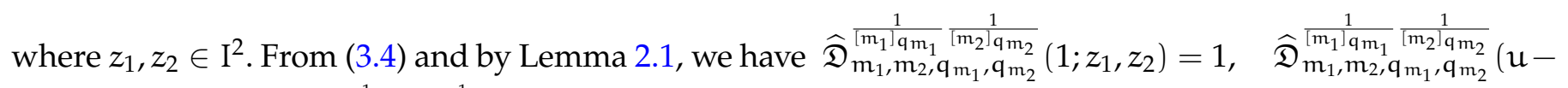

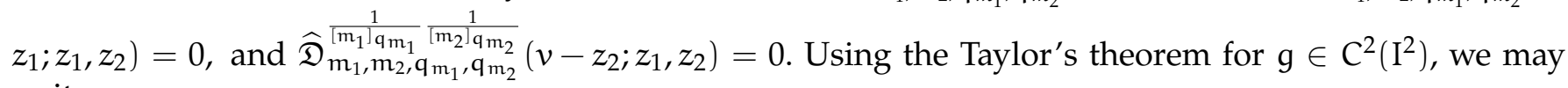
write

$$
\begin{aligned}
g(u, v)-g\left(z_{1}, z_{2}\right)= & \frac{\partial g\left(z_{1}, z_{2}\right)}{\partial z_{1}}\left(u-z_{1}\right)+\int_{z_{1}}^{u}(u-\eta) \frac{\partial^{2} g(\eta, y)}{\partial \eta^{2}} d \eta \\
& +\frac{\partial g\left(z_{1}, z_{2}\right)}{\partial z_{2}}\left(v-z_{2}\right)+\int_{z_{2}}^{v}(v-\xi) \frac{\partial^{2} g(x, \xi)}{\partial \xi^{2}} d \xi .
\end{aligned}
$$

Applying the operator $\widehat{\mathfrak{D}}_{\mathrm{m}_{1}, \mathrm{~m}_{2}, \mathrm{q}_{\mathrm{m}_{1}}, \mathrm{q}_{\mathrm{m}_{2}}}^{\frac{1}{\left[\mathrm{~m}_{2} \mathrm{q}_{\mathrm{m}_{2}}\right.}} \frac{1}{\mathrm{~m}_{\mathrm{m}}}$ on both sides of the above equation, we get

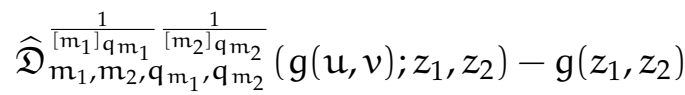

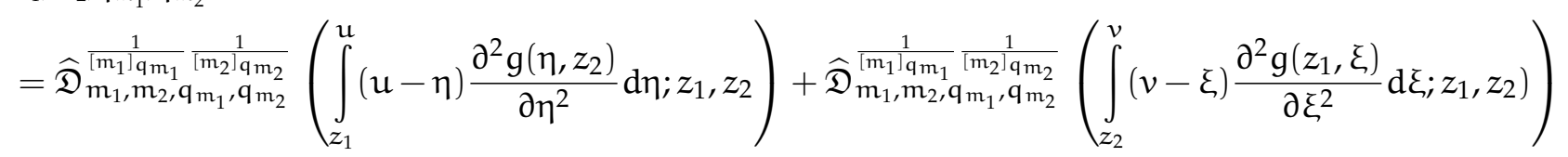

$$
\begin{aligned}
& \left.=\mathfrak{D}_{m_{1}, m_{2}, q_{m_{1}}, q_{m_{2}}}^{\frac{1}{\left[m_{1}\right] q_{m_{1}}}} \frac{1}{\left[m_{2}\right] m_{2}}\left(\int_{z_{1}}^{u}(u-\eta) \frac{\partial^{2} g\left(\eta, z_{2}\right)}{\partial \eta^{2}} d \eta ; z_{1}, z_{2}\right)\right)-\int_{z_{1}}^{u_{m_{1}}}\left(u_{m_{1}}-\eta\right) \frac{\partial^{2} g\left(z_{1}, \eta\right)}{\partial \eta^{2}} d \eta
\end{aligned}
$$




$$
\left.+\mathfrak{D}_{m_{1}, m_{2}, q_{m_{1}}, q_{m_{2}}}^{\frac{1}{\left[m_{1} q_{m_{1}}\right.}} \frac{1}{\left[m_{2}\right] q_{m_{2}}}\left(\int_{z_{2}}^{v}(v-\xi) \frac{\partial^{2} g\left(\xi, z_{1}\right)}{\partial \xi^{2}} \mathrm{~d} \xi ; z_{1}, z_{2}\right)\right)-\int_{z_{2}}^{v_{m_{2}}}\left(v_{m_{2}}-\xi\right) \frac{\partial^{2} g\left(z_{1}, \xi\right)}{\partial \xi^{2}} \mathrm{~d} \xi .
$$

On the other hand, since

$$
\begin{aligned}
\left|\int_{z_{1}}^{u}(u-\eta) \frac{\partial^{2} g\left(\eta, z_{2}\right)}{\partial \eta^{2}} d \eta\right| & \leqslant\left|\int_{z_{1}}^{u}\right|(u-\eta)\left|\frac{\partial^{2} g\left(\eta, z_{2}\right)}{\partial \eta^{2}}\right| d \eta \mid \\
& \leqslant\|g\|_{C^{2}\left(I^{2}\right)}\left|\int_{z_{1}}^{u}\right| u-\eta|| \frac{\partial^{2} g\left(\eta, z_{2}\right)}{\partial \eta^{2}}|d \eta| \leqslant\|g\|_{C^{2}\left(I^{2}\right)}\left(u-z_{2}\right)^{2},
\end{aligned}
$$

and analogously

$$
\left|\int_{z_{1}}^{u_{m_{1}}}\left(u_{m_{1}}-\eta\right) \frac{\partial^{2} g\left(\eta, z_{2}\right)}{\partial \eta^{2}} d \eta\right| \leqslant\left(u_{m_{1}}-z_{1}\right)^{2}\|g\|_{C^{2}\left(I^{2}\right)}
$$

we conclude that

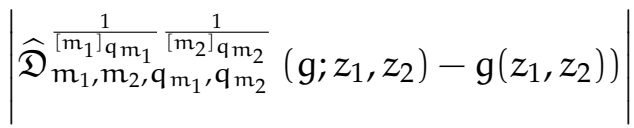

$$
\begin{aligned}
& \left.\leqslant \mathfrak{D}_{m_{1}, m_{2}, q_{m_{1}}, q_{m_{2}}}^{\frac{1}{\left[m_{1} ! q_{m_{1}}\right.} \frac{1}{\left(m_{2} ! q_{2}\right.}}\left(\left|\int_{z_{1}}^{u}(u-\eta) \frac{\partial^{2} g\left(\eta, z_{2}\right)}{\partial \eta^{2}} d \eta\right| ; z_{1}, z_{2}\right)\right) \\
& +\left|\int_{z_{1}}^{u_{m_{1}}}\left(u_{m_{1}}-\eta\right) \frac{\partial^{2} g\left(z_{1}, \eta\right)}{\partial \eta^{2}} d \eta\right|+\mathfrak{D}_{m_{1}, m_{2}, q_{m_{1}}, q_{m_{2}}}^{\frac{1}{\left[m_{1}\right] m_{m_{1}}} \frac{1}{\left[m_{2}\right] q_{m_{2}}}}\left(\left|\int_{z_{2}}^{v}(v-\xi) \frac{\partial^{2} g\left(\xi, z_{1}\right)}{\partial \xi^{2}} d \eta\right| ; z_{1}, z_{2}\right) \\
& \left.+\left|\int_{z_{2}}^{v_{m_{2}}}\left(v_{m_{2}}-\xi\right) \frac{\partial^{2} g\left(z_{1}, \xi\right)}{\partial \xi^{2}} d \xi\right| \leqslant\left\{\mathfrak{D}_{m_{1}, q_{m_{1}}}^{\frac{1}{m_{1} 1 q_{1}}}\left(\left(u-z_{1}\right)^{2} ; z_{1}, z_{2}\right)-\left(u_{m_{1}}-z_{1}\right)^{2}\right)\right\}\|g\|_{C_{\left(1^{2}\right)}^{2}} \\
& \left.+\left\{\mathfrak{D}_{\mathrm{m}_{2}, \mathbf{q}_{\mathrm{m}_{2}}}^{\frac{1}{\left[\mathrm{~m}_{2} \mid \mathbf{q}_{\mathrm{m}_{2}}\right.}}\left(\left(v-z_{2}\right)^{2} ; z_{1}, z_{2}\right)+\left(v_{\mathrm{m}_{2}}-z_{2}\right)^{2}\right)\right\}\|g\|_{\mathrm{C}_{\left(1^{2}\right)}^{2}} \\
& \leqslant\left\{\delta_{m_{1}}+\delta_{m_{2}}+\left(u_{m_{1}}-z_{1}\right)^{2}+\left(v_{m_{2}}-z_{2}\right)^{2}\right\}\|g\|_{C^{2}\left(I^{2}\right)} \\
& =\eta_{m_{1}, m_{2}}\left(h ; q_{m_{1}}, q_{m_{2}}, z_{1}, z_{2}\right)\|g\|_{C^{2}\left(I^{2}\right)} \text {. }
\end{aligned}
$$

On using Equation (3.4), we see that

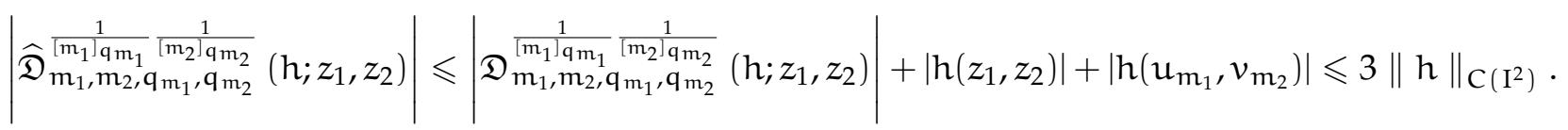

Hence in view of (3.6) and (3.7), we have

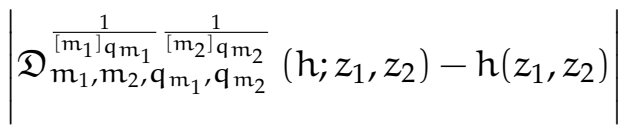

$$
\begin{aligned}
& =\left|\widehat{\mathfrak{D}} \frac{1}{\frac{1}{m_{m_{1}}, m_{2}, q_{m_{1}}, q_{m_{2}}} \frac{1}{\left[m_{2}\right] q_{2}}}\left(h ; z_{1}, z_{2}\right)-h\left(z_{1}, z_{2}\right)+h\left(u_{m_{1}}, v_{m_{2}}\right)-h\left(z_{1}, z_{2}\right)\right|
\end{aligned}
$$

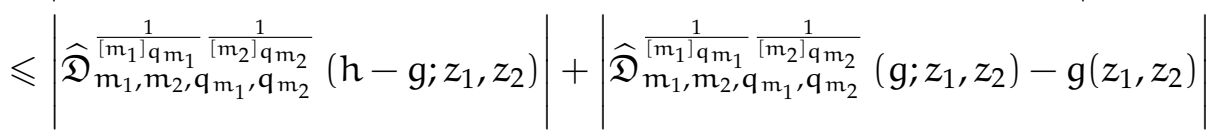




$$
\begin{aligned}
& +\left|g\left(z_{1}, z_{2}\right)-h\left(z_{1}, z_{2}\right)\right|+\left|h\left(u_{m_{1}}, v_{m_{2}}\right)-h\left(z_{1}, z_{2}\right)\right| \\
& \leqslant 4\|h-g\|_{C\left(I_{a b}\right)}+\left|\widehat{\mathfrak{D}}_{m_{1}, m_{2}, q_{m_{1}}, q_{m_{2}}}^{\frac{1}{\left[m_{1}\right] m_{m}} \frac{1}{\left.m_{2}\right] q_{m}}}\left(g ; z_{1}, z_{2}\right)-g\left(z_{1}, z_{2}\right)\right|+\left|h\left(u_{m_{1}}, v_{m_{2}}\right)-h\left(z_{1}, z_{2}\right)\right| \\
& \leqslant\left(4\|h-g\|_{C\left(I^{2}\right)}+\eta_{m_{1}, m_{2}}\left(h ; q_{m_{1}}, q_{m_{2}}, z_{1}, z_{2}\right)\right)\|g\|_{C\left(I^{2}\right)}+\omega\left(h ; \sqrt{\left(u_{m_{1}}-z_{1}\right)^{2}+\left(v_{m_{2}}-z_{2}\right)^{2}}\right) \\
& \leqslant 4 K\left(h ; \eta_{m_{1}, m_{2}, p_{1}, p_{2}}\left(q_{m_{1}}, q_{m_{2}}, z_{1}, z_{2}\right)\right)+\omega\left(h ; \sqrt{\left(u_{m_{1}}-z_{1}\right)^{2}+\left(v_{m_{2}}-z_{2}\right)^{2}}\right) \\
& \leqslant L\left\{\omega_{2}\left(h ; \sqrt{\eta_{m_{1}, m_{2}, p_{1}, p_{2}}\left(q_{m_{1}}, q_{m_{2}}, z_{1}, z_{2}\right)}\right)\right. \\
& \left.+\min \left\{1, \eta_{m_{1}, m_{2}, p_{1}, p_{2}}\left(q_{m_{1}}, q_{m_{2}}, z_{1}, z_{2}\right)\right\}\|h\|_{C_{\left(I^{2}\right)}}\right\}+\omega\left(h ; \sqrt{\left(u_{m_{1}}-z_{1}\right)^{2}+\left(v_{m_{2}}-z_{2}\right)^{2}}\right) .
\end{aligned}
$$

This completes the proof.

Theorem 3.7. If $\mathrm{h}\left(z_{1}, z_{2}\right)$ has continuous partial derivatives $\frac{\partial \mathrm{h}}{\partial z_{1}}$ and $\frac{\partial \mathrm{h}}{\partial z_{2}}$, as well as $\omega^{(1)}\left(\mathrm{h}_{z_{1}}^{\prime} ; \delta\right)$ and $\omega^{(2)}\left(\mathrm{h}_{z_{2}}^{\prime} ; \delta\right)$ denotes the partial modules of continuity of $\frac{\partial \mathrm{h}}{\partial z_{1}}$ and $\frac{\partial \mathrm{h}}{\partial z_{2}}$, respectively, then holds the inequality

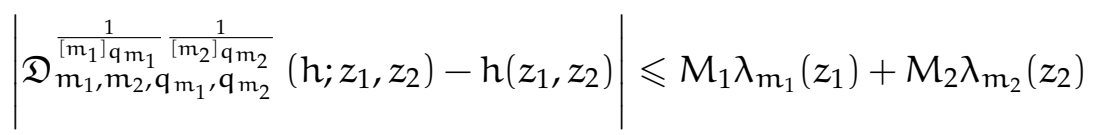

$$
\begin{aligned}
& +\omega^{(1)}\left(h_{z_{1}}^{\prime} ; \delta_{m_{1}}\right)\left(1+\sqrt{\delta_{m_{1}}}\right)+\omega^{(2)}\left(h_{z_{1}}^{\prime} ; \delta_{m_{2}}\right)\left(1+\sqrt{\delta_{m_{2}}}\right),
\end{aligned}
$$

where $M_{1}, M_{2}$ are the positive constants such that $\left|\frac{\partial h}{\partial z_{1}}\right| \leqslant M_{1},\left|\frac{\partial h}{\partial z_{1}}\right| \leqslant M_{2},\left(0 \leqslant z_{1} \leqslant a, 0 \leqslant z_{2} \leqslant b\right)$, and

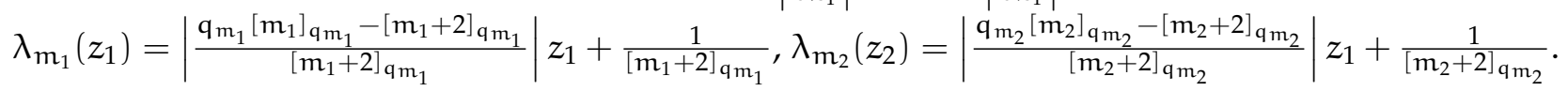

Proof. From the mean value theorem we have

$$
\begin{aligned}
h\left(t_{1}, t_{2}\right)-h\left(z_{1}, z_{2}\right)= & h\left(t_{1}, z_{2}\right)-h\left(z_{1}, z_{2}\right)+h\left(t_{1}, t_{2}\right)-h\left(t_{1}, z_{2}\right) \\
= & \left(t_{1}-z_{1}\right) \frac{\partial h\left(\xi_{1}, z_{2}\right)}{\partial z_{1}}+\left(t_{2}-z_{2}\right) \frac{\partial h\left(z_{1}, \xi_{2}\right)}{\partial z_{2}} \\
= & \left(t_{1}-z_{1}\right) \frac{\partial h\left(z_{1}, z_{2}\right)}{\partial z_{1}}+\left(t_{1}-z_{1}\right)\left(\frac{\partial h\left(\xi_{1}, z_{2}\right)}{\partial z_{1}}-\frac{\partial h\left(z_{1}, z_{2}\right)}{\partial z_{1}}\right) \\
& +\left(t_{2}-z_{2}\right) \frac{\partial h\left(z_{1}, z_{2}\right)}{\partial z_{2}}+\left(t_{2}-z_{2}\right)\left(\frac{\partial h\left(z_{1}, \xi_{2}\right)}{\partial z_{2}}-\frac{\partial h\left(z_{1}, z_{2}\right)}{\partial z_{2}}\right),
\end{aligned}
$$

where $z_{1}<\xi_{1}<t_{1}$ and $z_{2}<\xi_{2}<t_{2}$. By using the above identity, we get

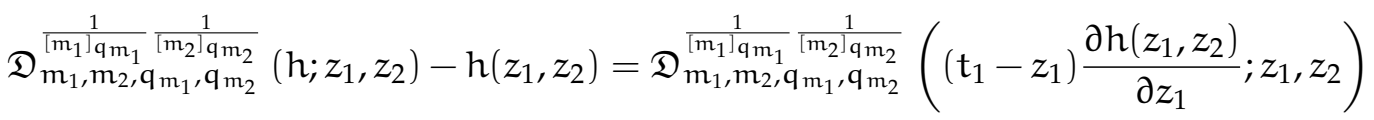

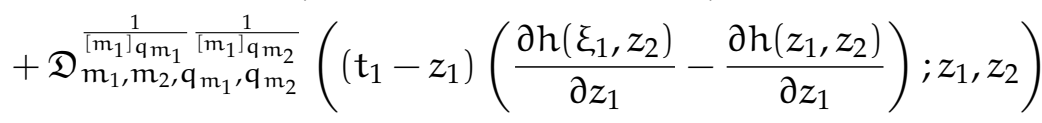

$$
\begin{aligned}
& \left.+\mathfrak{D}_{\mathrm{m}_{1}, \mathrm{~m}_{2}, \mathbf{q}_{\mathrm{m}_{1}}, \mathbf{q}_{\mathrm{m}_{2}}}^{\frac{1}{\left[\mathrm{~m}_{2}\right]}} \frac{1}{\left[\mathrm{~m}_{2}\right] \mathrm{m}_{2}}\left(\mathrm{t}_{2}-z_{2}\right) \frac{\partial \mathrm{h}\left(z_{1}, z_{2}\right)}{\partial z_{2}} ; z_{1}, z_{2}\right)
\end{aligned}
$$

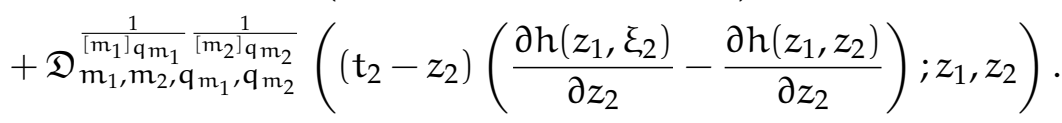


Hence,

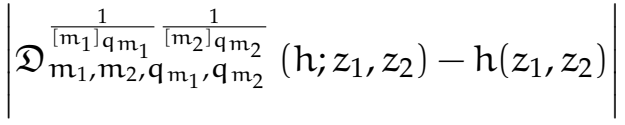

$$
\begin{aligned}
& \leqslant\left|\frac{\partial h\left(z_{1}, z_{2}\right)}{\partial z_{1}}\right|\left|\mathfrak{D}_{m_{1}, m_{2}, q_{m_{1}}, q_{m_{2}}}^{\frac{1}{\left[m_{1}\right] q_{m_{2}}} \frac{1}{\left[m_{2} q_{m_{2}}\right.}}\left(\left(t_{1}-z_{1}\right) ; z_{1}, z_{2}\right)\right|
\end{aligned}
$$

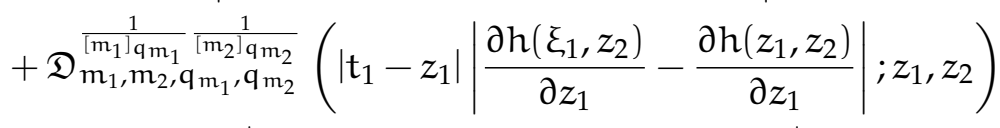

$$
\begin{aligned}
& +\left|\frac{\partial h\left(z_{1}, z_{2}\right)}{\partial z_{2}}\right|\left|\mathfrak{D}_{m_{1}, m_{2}, q_{m_{1}}, q_{m_{2}}}^{\frac{1}{\left[m_{1}\right] m_{2}} \frac{1}{\left[m_{2}\right] m_{2}}}\left(\left(t_{2}-z_{2}\right) ; z_{1}, z_{2}\right)\right|
\end{aligned}
$$

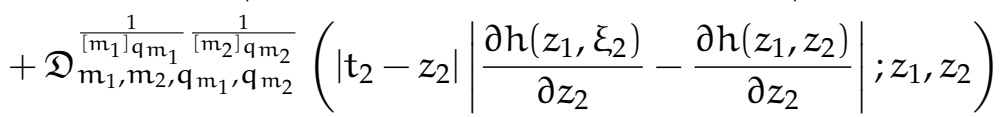

$$
\begin{aligned}
& \leqslant M_{1}\left|\mathfrak{D}_{m_{1}, m_{2}, q_{m_{1}}, q_{m_{2}}}^{\frac{1}{\left[m_{1} q_{m_{1}}\right.}} \frac{1}{\left[m_{2}\right] q_{m_{2}}}\left(\left(t_{1}-z_{1}\right) ; z_{1}, z_{2}\right)\right|+M_{2}\left|\mathfrak{D}_{m_{1}, m_{2}, q_{m_{1}}, q_{m_{2}}}^{\frac{1}{\left[m_{1} q_{m_{1}}\right.}} \frac{1}{\left[m_{2}\right] q_{m_{2}}}\left(\left(t_{2}-z_{2}\right) ; z_{1}, z_{2}\right)\right| \\
& +\mathfrak{D}_{\mathrm{m}_{1}, \mathrm{~m}_{2}, \mathbf{q}_{\mathrm{m}_{1}}, \mathbf{q}_{\mathrm{m}_{2}}}^{\frac{1}{\left[\mathrm{~m}_{1}\right]}}\left(\left|\mathrm{t}_{1}-z_{1}\right| \omega^{(1)}\left(\mathrm{h}_{z_{1}}^{\prime} ; \delta_{\mathrm{m}_{1}}\right)\left(\frac{\left|\mathrm{t}_{1}-z_{1}\right|}{\delta_{\mathrm{m}_{1}}}+1\right) ; z_{1}, z_{2}\right)
\end{aligned}
$$

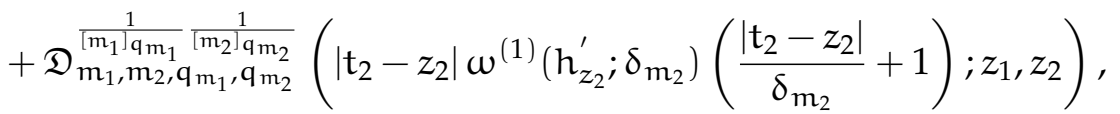

since $\left|\xi_{1}-z_{1}\right|<\left|t_{1}-z_{1}\right|$ and $\left|\xi_{2}-z_{2}\right|<\left|t_{2}-z_{2}\right|$. Using last inequalities, we have

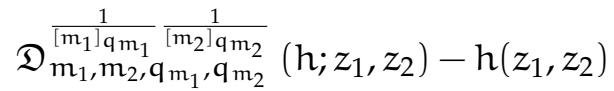

$$
\begin{aligned}
& \leqslant M_{1}\left|\mathfrak{D}_{m_{1}, m_{2}, q_{m_{1}}, q_{m_{2}}}^{\frac{1}{\left[m_{1}\right] m_{1}}} \frac{1}{\left[m_{2}\right] m_{2}}\left(\left(t_{1}-z_{1}\right) ; z_{1}, z_{2}\right)\right|
\end{aligned}
$$

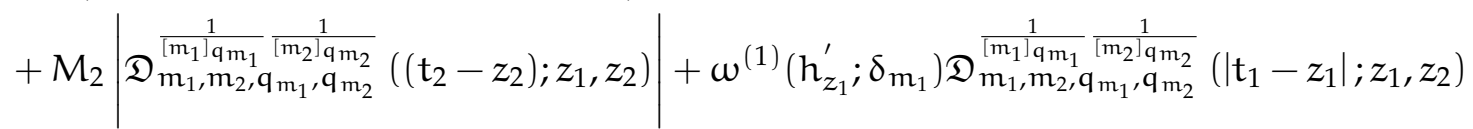

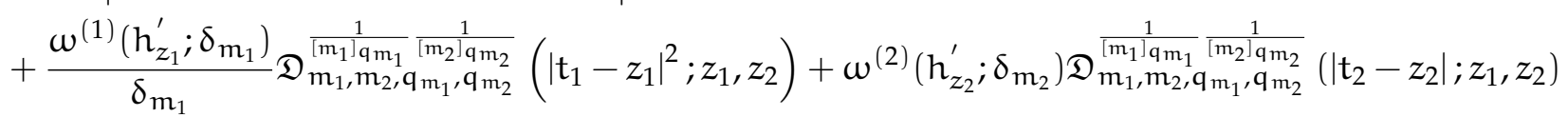

$$
\begin{aligned}
& +\frac{\omega^{(2)}\left(\mathrm{h}_{z_{2}}^{\prime} ; \delta_{\mathrm{m}_{2}}\right)}{\delta_{\mathrm{m}_{2}}} \mathfrak{D}_{\mathrm{m}_{1}, \mathrm{~m}_{2}, \mathbf{q}_{\mathrm{m}_{1}}, \mathbf{q}_{\mathrm{m}_{2}}}^{\frac{1}{\mathrm{~m}_{1} \mathrm{q}_{\mathrm{m}_{2}}}} \frac{1}{\frac{1}{m_{2} \mathrm{q}_{\mathrm{m}_{2}}}}\left(\left|\mathrm{t}_{2}-z_{2}\right|^{2} ; z_{1}, z_{2}\right) \text {. }
\end{aligned}
$$

Now, applying the Cauchy-Schwarz inequality

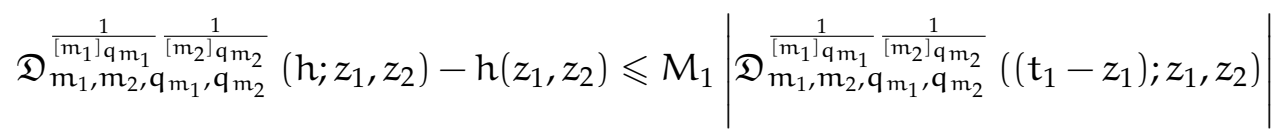

$$
\begin{aligned}
& +M_{2}\left|\mathfrak{D}_{m_{1}, m_{2}, q_{m_{1}}, q_{m_{2}}}^{\frac{1}{\left[m_{1} q_{m_{1}}\right.}} \frac{1}{\left[m_{2}\right]}\left(\left(t_{2}-z_{2}\right) ; z_{1}, z_{2}\right)\right| \\
& +w^{(1)}\left(h_{z_{1}}^{\prime} ; \delta_{m_{1}}\right)\left\{\mathfrak{D}_{m_{1}, q_{m_{1}}}^{\frac{1}{\left[m_{1} \mathfrak{q}_{m_{1}}\right.}}\left(\left(\mathrm{t}_{1}-z_{1}\right)^{2} ; z_{1}, z_{2}\right)\right\}^{1 / 2} \\
& +\frac{\omega^{(1)}\left(h_{z_{1}}^{\prime} ; \delta_{m_{1}}\right)}{\delta_{m_{1}}} \mathfrak{D}_{m_{1}, q_{m_{1}}}^{\frac{1}{m_{1} q_{m_{1}}}}\left(\left(t_{1}-z_{1}\right)^{2} ; z_{1}, z_{2}\right) \\
& +w^{(2)}\left(h_{z_{2}}^{\prime} ; \delta_{m_{2}}\right)\left\{\mathfrak{D}_{m_{2}, q_{m_{2}}}^{\frac{1}{\left[m_{2} q_{m_{2}}\right.}}\left(\left(t_{2}-z_{2}\right)^{2} ; z_{1}, z_{2}\right)\right\}^{1 / 2}
\end{aligned}
$$




$$
+\frac{\omega^{(2)}\left(h_{z_{2}}^{\prime} ; \delta_{m_{2}}\right)}{\delta_{m_{2}}} \mathfrak{D}_{m_{2}, q_{m_{2}}}^{\frac{1}{\left[m_{2}\right] q_{m_{2}}}}\left(\left(t_{2}-z_{2}\right)^{2} ; z_{1}, z_{2}\right) .
$$

By choosing $\delta_{\mathrm{m}_{1}}=\delta_{\mathrm{m}_{1}}\left(z_{1}\right)$ and $\delta_{\mathrm{m}_{2}}=\delta_{\mathrm{m}_{2}}\left(z_{2}\right)$, we have

$$
\begin{aligned}
& \left|\mathfrak{D}_{\mathrm{m}_{1}, \mathrm{~m}_{2}, \mathbf{q}_{\mathrm{m}_{1}}, \mathbf{q}_{\mathrm{m}_{2}}}^{\frac{1}{\left[\mathrm{~m}_{2}\right] \mathrm{q}_{\mathrm{m}_{2}}}}\left(\mathrm{~h} ; z_{1}, z_{2}\right)-\mathrm{h}\left(z_{1}, z_{2}\right)\right| \leqslant M_{1} \lambda_{\mathrm{m}_{1}}\left(z_{1}\right)+M_{2} \lambda_{\mathrm{m}_{2}}\left(z_{2}\right) \\
& +\omega^{(1)}\left(h_{z_{1}}^{\prime} ; \delta_{m_{1}}\right)\left(1+\sqrt{\delta_{m_{1}}}\right)+\omega^{(2)}\left(h_{z_{2}}^{\prime} ; \delta_{m_{2}}\right)\left(1+\sqrt{\delta_{m_{2}}}\right) .
\end{aligned}
$$

This completes the proof.

\section{References}

[1] P. N. Agrawal, B. Baxhaku, R. Chauhan, The approximation of bivariate Chlodowsky-Szász-Kantorovich-Charlier-type operators, J. Inequal. Appl., 2017 (2017), 23 pages. 1

[2] A. Aral, V. Gupta, On the q analogue of Stancu-beta operators, Appl. Math. Lett., 25 (2012), 67-71. 1

[3] A. Aral, V. Gupta, R. P. Agarwal, Applications of q-Calculus in Operator Theory, Springer, New York, (2013). 1

[4] D. Bărbosu, C. V. Muraru, A.-M. Acu, Some bivariate Durrmeyer operators based on q-integers, J. Math. Inequal., 11 (2017), 59-75. 1, ii

[5] B. Baxhaku, P. N. Agrawal, Degree of approximation for bivariate extension of Chlodowsky-type q-Bernstein-StancuKantorovich operators, Appl. Math. Comput., 306 (2017), 56-72. 1

[6] P. L. Butzer, H. Berens, Semi-groups of operators and approximation, Springer, Berlin, (2013). 3

[7] O. Doğru, V. Gupta, Monotonicity and the asymptotic estimate of Bleimann Butzer and Hahn operators based on q-integers, Georgian Math. J., 12 (2005), 415-422. 1

[8] V. Gupta, Some approximation properties of q-Durrmeyer operators, Appl. Math. Comput., 197 (2008), 172-178. 1

[9] V. Gupta, A. Aral, Bernstein Durrmeyer operators based on two parameters, Facta Univ. Ser. Math. Inform., 31 (2016), 79-95. 1

[10] V. Gupta, T. M. Rassias, H. Sharma, q-Durrmeyer operators based on Pólya distribution, J. Nonlinear Sci. Appl., 9 (2016), 1497-1504. 1, 1.2, 2

[11] N. İspir, İ. Büyükyazıcı, Quantitative estimates for a certain bivariate Chlodowsky-Szasz-Kantorovich type operators, Math. Commun., 21 (2016), 31-44. 1

[12] A. Lupaş, A q-analogue of the Bernstein operator, Seminar on Numerical and Statistical Calculus, Univ. "BabeşBolyai", Cluj-Napoca, (1987), 85-92. 1

[13] C. V. Muraru, A. M. Acu, Some approximation properties of q-Durrmeyer-Schurer operators, Sci. Stud. Res. Ser. Math. Inform., 23 (2013), 77-84. 1

[14] G. Nowak, Approximation properties for generalized q-Bernstein polynomials, J. Math. Anal. Appl., 350 (2009), 50-55. $1,1, \mathrm{i}$

[15] G. M. Phillips, Bernstein polynomials based on the q-integers, Ann. Numer. Math., 4 (1997), 511-518. 1

[16] R. Ruchi, B. Baxhaku, P. N. Agrawal, GBS operators of bivariate Bernstein-Durrmeyer-type on a triangle, Math. Methods Appl. Sci., 41 (2018), 2673-2683. 1

[17] D. D. Stancu, Approximation of functions by a new class of linear polynomial operators, Rev. Roumaine Math. Pures Appl., 13 (1968), 1173-1194. 1, 1

[18] I. V. Volkov, On the convergence of a sequence of linear positive operators in the space of continuous funtions of two variables, Dokl. Akad. Nauk SSSR, 115 (1957), 17-19. 3 